\title{
Effects of Reservoir Heterogeneity on Scaling of Effective Mass Transfer Coefficient for Solute Transport
}

\author{
Juliana Y. Leung ${ }^{a^{*}}$, Sanjay Srinivasan ${ }^{\mathrm{b}+}$ \\ ${ }^{a}$ Department of Civil and Environmental Engineering, School of Mining and Petroleum Engineering, University of Alberta, Canada \\ ${ }^{b}$ Department of Petroleum and Geosystems Engineering, University of Texas at Austin, USA
}

\begin{abstract}
Modeling transport process at large scale requires proper scale-up of subsurface heterogeneity and an understanding of its interaction with the underlying transport mechanisms. A technique based on volume averaging is applied to quantitatively assess the scaling characteristics of effective mass transfer coefficient in heterogeneous reservoir models. The effective mass transfer coefficient represents the combined contribution from diffusion and dispersion to the transport of non-reactive solute particles within a fluid phase. Although treatment of transport problems with the volume averaging technique has been published in the past, application to geological systems exhibiting realistic spatial variability remains a challenge. Previously, the authors developed a new procedure where results from a fine-scale numerical flow simulation reflecting the full physics of the transport process albeit over a sub-volume of the reservoir are integrated with the volume averaging technique to provide effective description of transport properties. The procedure is extended such that spatial averaging is performed at the local-heterogeneity scale.

In this paper, the transport of a passive (non-reactive) solute is simulated on multiple reservoir models exhibiting different patterns of heterogeneities, and the scaling behavior of effective mass transfer coefficient $\left(K_{e f f}\right)$ is examined and compared. One such set of models exhibit power-

*Corresponding author: Phone: +1 7804923338

Mailing address: 6-285 Donadeo Innovation Centre for Engineering, 9211 116th Street, Edmonton, Alberta, Canada T6H 1G9

E-mail address: juliana2@ualberta.ca.

${ }^{+}$Now with John and Willie Leone Family Department of Energy and Mineral Engineering, Pennsylvania State University
\end{abstract}

(C) 2016. This manuscript version is made available under the Elsevier user license

http://www.elsevier.com/open-access/userlicense/1.0/ 
law (fractal) characteristics, and the variability of dispersion and $K_{\text {eff }}$ with scale is in good agreement with analytical expressions described in the literature.

This work offers an insight into the impacts of heterogeneity on the scaling of effective transport parameters. A key finding is that spatial heterogeneity models with similar univariate and bivariate statistics may exhibit different scaling characteristics because of the influence of higher order statistics. More mixing is observed in the channelized models with higher-order continuity. It reinforces the notion that the flow response is influenced by the higher-order statistical description of heterogeneity. An important implication is that when scaling-up transport response from lab-scale results to the field scale, it is necessary to account for the scaleup of heterogeneity. Since the characteristics of higher-order multivariate distributions and largescale heterogeneity are typically not captured in small-scale experiments, a reservoir modeling framework that captures the uncertainty in heterogeneity description should be adopted.

Keywords: effective mass transfer coefficient; scaling methods; volume averaging; fractals; geostatistics

\section{Introduction}

There are a number of methods that can be employed to understand the scale-up characteristics of transport and flow in geologic media. A simple approach is to correlate dimensionless groups depicting the relative magnitudes of different transport mechanisms with various heterogeneity scaling factors (Li \& Lake, 1995). These heterogeneity factors may include a combination of statistical measures related to the permeability distribution (e.g., variance or a specific quantile). The major drawback with this approach is that detailed description regarding 
spatial variability of reservoir parameters cannot be readily incorporated into these dimensionless groups.

The second approach is to evaluate the scale-up characteristics by deriving the appropriate transport equations at the macroscopic scale as averages of fine-scale quantities. The two main classes of such techniques are 1) Ensemble Averaging Method (Cvetkovic \& Gotovac, 2014; Fathi \& Akkutlu, 2012; Zhang \& Winter, 1999), 2) Spatial Moment Analysis (Brenner, 1980a \& 1980b) and 3) Volume Averaging Method (Whitaker, 1999). In ensemble averaging, the mean state and the variance around the mean state of a system at the larger scale are estimated over an ensemble of realizations. This variance is attributed to the incomplete knowledge of the exact structure of the small-scale heterogeneity. If a random field is ergodic, ensemble average converges to spatial average. In contrast to the method of ensemble averaging, both spatial moment and volume averaging utilizes spatial averages of a random field. The spatial moment method assumes that the distribution of scaled-up transport response can be characterized knowing spatial moments; therefore, expressions for the asymptotic effective parameters can be obtained via the generalized moment analysis (Chrysikopoulos et al., 1992). Local-scale variability is assumed to be known and often described as periodic (Chrysikopoulos, 1995). However, computation of spatial moments for arbitrarily heterogeneous media is not well understood.

For the volume averaging method, fine-scale transport equations are spatially averaged to produce continuum equations that are valid everywhere in the system. A coupled set of largescale average and small-scale deviation equations are formulated based on the concept of spatial stationarity (Wood, 2013). Since heterogeneity influences the spatial mean, the concepts of volume averaging, as compared to ensemble averaging, more closely conform to our objectives. 
This method is particularly useful for deriving statistics (mean or variance) of effective transport parameters (provided that reservoir heterogeneity is accounted for) as a function of length scale. Therefore, it is possible to incorporate arbitrarily complex heterogeneity in the procedure. Many researchers have used this method in the past to model flow and transport characteristics in porous media. General treatments of the volume averaging techniques can be found in Whitaker (1999) and Wood (2009). The most common types of transport problems studied are solute transport (Zanotti \& Carbonell, 1984) and fluid flow (Quintard \& Whitaker, 1988). Scaled-up versions of various transport parameters such as dispersivity, effective mass transfer coefficient, and permeability tensor were derived. Specific applications relating to reaction-transport processes can be found in and Kechagia et al. (2002), Coutelieris et al. (2006), Porta et al. (2013) and Lugo-Méndez et al. (2015).

In the conventional implementation of volume averaging, numerous assumptions (e.g. linear averaging of velocities) are invoked to render the formulations tractable for implementation. These assumptions limit its application to mostly simple descriptions of spatial heterogeneity such as a periodic medium. In Leung \& Srinivasan (2012), a procedure where results from a finescale numerical flow simulation performed over a small sub-volume of the reservoir is integrated. The fine-scale simulation can be performed on arbitrarily complex heterogeneous media. The authors have presented details about the implementation and application of the method to both single and multi-phase systems in the past (Leung \& Srinivasan, 2011; 2012, Leung, 2014). Formulations for averaging from the pore scale to the continuum (Darcy) scale were presented in Leung \& Srinivasan (2012). It was mentioned that a similar procedure can be applied for averaging from the continuum (Darcy) to the scale of local heterogeneity characterized by lithofacies. Using fine-scale properties defined at the continuum scale for each 
lithofacies. The final forms of the volume-averaged equations are identical except for different interfacial or inter-regional integral terms that are evaluated together using an interfacial parameter $\alpha$. In this paper, the formulation and solution of effective properties described on the basis of lithofacies distribution at the local heterogeneity scale is discussed. This development renders it feasible to derive scaled up parameters for solute transport processes where the transport at the fine scale is controlled by the spatial arrangement of distinct facies - a situation that best describes transport in most complex geologic systems.

This work presents a systematic approach for quantifying the variation and uncertainty of effective mass transfer coefficient with scale in a given heterogeneous reservoir. The volume averaging approach is modified by utilizing information from numerical fine-scale flow simulations and integrating heterogeneity into the scale-up of transport equations from Darcy scale to field scale. The case studies highlight how the continuity of spatially-correlated properties, as characterized by the higher-order multivariate statistics, may influence the uncertainty in the coarse-scale properties. In many instances, the impacts of higher-order multivariate distributions and large-scale heterogeneity could not be possibly captured in smallscale physical experiments of the transport process; the results suggest uncertainty in random function modeling of reservoir properties would introduce uncertainty in the scaling of effective transport parameters. Therefore, ignoring their impacts could severely underestimate the ensuing uncertainties in transport behavior at the field scale.

In the subsequent sections, the governing equations are presented, followed by a discussion of the averaging process and the implementation details. Finally, the method is applied on several case studies where reservoir properties are characterized by random function models constrained to varying statistical description of heterogeneities - covariance constrained models compared to 
channelized models constrained to multiple-point statistics, and power law (fractal) models. The paper concludes with a discussion on the influence of heterogeneity on the scale-up characteristics of solute transport processes.

\section{Mathematical Formulation}

Following the notations in Ahmadi et al. (1998) and Quintard \& Whitaker (1998), we begin with the governing equations that describe solute transport within a single incompressible flowing phase at Darcy (continuum) scale and isothermal conditions (Leung \& Srinivasan, 2011; 2012).

$$
\begin{aligned}
& \varepsilon_{\eta} \frac{\partial\langle C\rangle^{\eta}}{\partial t}+\nabla \cdot\left(\langle\mathbf{u}\rangle_{\eta}\langle C\rangle^{\eta}\right)=\nabla \cdot\left(\varepsilon_{\eta} \mathbf{D}_{\eta} \cdot \nabla\langle C\rangle^{\eta}\right) \\
& \varepsilon_{\sigma} \frac{\partial\langle C\rangle^{\sigma}}{\partial t}+\nabla \cdot\left(\langle\mathbf{u}\rangle_{\varpi}\langle C\rangle^{\varpi}\right)=\nabla \cdot\left(\varepsilon_{\varpi} \mathbf{D}_{\varpi} \cdot \nabla\langle C\rangle^{\sigma}\right)
\end{aligned}
$$

B.C. 1: $\langle C\rangle^{\eta}=\langle C\rangle^{\sigma}$ at $A_{\eta \omega}$

B.C. 2: $-\mathbf{n}_{\eta \sigma} \cdot\left(\langle\mathbf{u}\rangle_{\eta}\langle C\rangle^{\eta}-\varepsilon_{\eta} \mathbf{D}_{\eta} \cdot \nabla\langle C\rangle^{\eta}\right)=\mathbf{n}_{\sigma \eta} \cdot\left(\langle\mathbf{u}\rangle_{\sigma}\langle C\rangle^{\sigma}-\varepsilon_{\sigma} \mathbf{D}_{\sigma} \cdot \nabla\langle C\rangle^{\sigma}\right)$ at $A_{\eta \omega}$

The notations $\omega$ and $\eta$ represent different constituent regions of local heterogeneity (e.g., lithofacies) with length scales $l_{\omega}$ and $l_{\eta}$ in an averaging volume $V_{\infty}$. The quantities $\langle\mathbf{u}\rangle_{\varpi},\langle\mathbf{u}\rangle_{\eta}$, $\langle C\rangle^{\eta}$, and $\langle C\rangle^{\sigma}$ represent the superficial average velocity and intrinsic average concentration of a certain component at the local (Darcy) continuum scale. Following the commonly-adopted notation conventions, the angle brackets suggest that these quantities are defined at the local continuum scale, and that they represent averages of quantities defined at the pore scale. This formulation suggests that $l_{\omega}$ and $l_{\eta}$ should be greater than the length scale at which the continuum 
definition is considered valid. $\mathbf{D}_{\omega}$ and $\mathbf{D}_{\eta}$ refer to the effective dispersion tensors for the corresponding regions; $\varepsilon_{\omega}, \varepsilon_{\eta}$ are the total porosities in the $\omega$ or $\eta$-region, while $\mathbf{n}_{\omega \eta}$ is the normal vector to the area of boundary between the $\omega$ and $\eta$-regions $\left(A_{\omega \eta}\right)$. Although the equations for two local heterogeneity regions are derived, the formulation can be readily extended to include many regions. Eq. 1 describes the governing equations at the fine scale. Next the spatial averaging theorem (Cushman, 1982; Howes \& Whitaker, 1985) is used to derive a superficial volume average \{\} of the Eq. 1 that describes the coarse-scale transport behavior, where the curly brackets denote coarse-scale quantities In particular, for region $\omega$ :

$$
\begin{aligned}
& \varepsilon_{\sigma} \frac{\partial\left\{\langle C\rangle^{\sigma}\right\}}{\partial t}+\nabla \cdot\left\{\langle\mathbf{u}\rangle_{\varpi}\langle C\rangle^{\sigma}\right\}+\frac{1}{V_{\infty}} \int_{A_{\varpi \eta}} \mathbf{n}_{\varpi \eta} \cdot\left(\langle\mathbf{u}\rangle_{\varpi}\langle C\rangle^{\sigma}\right) d A \\
& =\nabla \cdot\left\{\varepsilon_{\sigma} \mathbf{D}_{\sigma} \cdot \nabla\langle C\rangle^{\sigma}\right\}+\frac{1}{V_{\infty}} \int_{A_{\varpi \eta}} \mathbf{n}_{\varpi \eta} \cdot\left(\varepsilon_{\sigma} \mathbf{D}_{\sigma} \cdot \nabla\langle C\rangle^{\sigma}\right) d A
\end{aligned}
$$

Assuming a constant dispersivity is defined at the fine scale, variations of dispersivity within the averaging volume $\left(V_{\infty}\right)$ can be ignored, the spatially-averaged equation becomes:

$$
\begin{aligned}
& \varepsilon_{\varpi} \frac{\partial\left\{\langle C\rangle^{\varpi}\right\}}{\partial t}+\nabla \cdot\left\{\langle\mathbf{u}\rangle_{\varpi}\langle C\rangle^{\varpi}\right\}+\frac{1}{V_{\infty}} \int_{A_{\varpi \eta}} \mathbf{n}_{\varpi \eta} \cdot\left(\langle\mathbf{u}\rangle_{\varpi}\langle C\rangle^{\varpi}\right) d A \\
& =\nabla \cdot\left\{\varepsilon_{\varpi} \mathbf{D}_{\varpi} \cdot\left(\nabla\left\{\langle C\rangle^{\sigma}\right\}+\frac{1}{V_{\infty}} \int_{A_{\varpi \eta}} \mathbf{n}_{\sigma \eta}\langle C\rangle^{\sigma} d A\right)\right\}+\frac{1}{V_{\infty}} \int_{A_{\varpi \eta}} \mathbf{n}_{\varpi \eta} \cdot\left(\varepsilon_{\varpi} \mathbf{D}_{\varpi} \cdot \nabla\langle C\rangle^{\sigma}\right) d A
\end{aligned}
$$

Fine-scale quantities, such as concentrations and velocities, can be expressed as a sum of their intrinsic average and a random fluctuation: $\langle C\rangle^{\sigma}=\left\{\langle C\rangle^{\sigma}\right\}^{\sigma}+C_{\sigma}^{\prime}$ where $\left\{\langle C\rangle^{\sigma}\right\}=\varphi_{\sigma}\left\{\langle C\rangle^{\sigma}\right\}^{\sigma}$ refers to the superficial average; $\varphi_{\omega},=$ volume fraction of region $\omega$ and $\langle\mathbf{u}\rangle_{\sigma}=\left\{\langle\mathbf{u}\rangle_{\sigma}\right\}^{\varpi}+\mathbf{u}_{\sigma}^{\prime}$. Assuming that all deviation quantities sum to zero over the average volume, that the fraction of each rock type remains constant throughout the domain and that the fluids are incompressible: 


$$
\begin{aligned}
& \frac{\partial \varphi_{\varpi}}{\partial t}=0 \\
& \nabla \cdot\left\{\langle\mathbf{u}\rangle_{\varpi}\right\}^{\varpi}=0
\end{aligned}
$$

The averaged equation for region $\omega$ becomes:

$$
\begin{aligned}
& \underbrace{\varepsilon_{\varpi} \varphi_{\varpi} \frac{\partial\left\{\langle C\rangle^{\varpi}\right\}^{\sigma}}{\partial t}}_{\text {accum }}+\underbrace{\nabla \cdot\left(\varphi_{\varpi}\left\{\mathbf{u}_{\varpi}^{\prime} C_{\varpi}^{\prime}\right\}^{\varpi}\right)}_{\text {dispersion }}+\underbrace{\varphi_{\varpi}\left\{\langle\mathbf{u}\rangle_{\varpi}\right\}^{\varpi} \cdot \nabla\left\{\langle C\rangle^{\sigma}\right\}^{\varpi}}_{\text {convection }}= \\
& \underbrace{\nabla \cdot\left[\varepsilon_{\varpi} \mathbf{D}_{\varpi} \cdot\left(\varphi_{\varpi} \nabla\left\langle\langle C\rangle^{\varpi}\right\}^{\varpi}+\frac{1}{V_{\infty}} \int_{A_{\varpi \eta}} \mathbf{n}_{\varpi \eta} C_{\bar{\sigma}}^{\prime} d A\right)\right]}_{\text {large-scale diffusion }} \underbrace{-\frac{1}{V_{\infty}} \int_{A_{\varpi \eta}} \mathbf{n}_{\varpi \eta} \cdot\left(\langle\mathbf{u}\rangle_{\varpi}\langle C\rangle^{\varpi}-\varepsilon_{\varpi} \mathbf{D}_{\varpi} \cdot \nabla\langle C\rangle^{\sigma}\right) d A}_{\text {inter-region flux }}
\end{aligned}
$$

Similarly, the averaged equation for region $\eta$ becomes:

$$
\begin{aligned}
& \varepsilon_{\eta} \varphi_{\eta} \frac{\partial\left\{\langle C\rangle^{\eta}\right\}^{\eta}}{\partial t}+\nabla \cdot\left(\varphi_{\eta}\left\{\mathbf{u}_{\eta}^{\prime} C_{\eta}^{\prime}\right\}^{\eta}\right)+\varphi_{\eta}\left\{\langle\mathbf{u}\rangle_{\eta}\right\} \cdot \nabla\left\{\langle C\rangle^{\eta}\right\}^{\eta}= \\
& \nabla \cdot\left[\varepsilon_{\eta} \mathbf{D}_{\eta} \cdot\left(\varphi_{\eta} \nabla\left\{\langle C\rangle^{\eta}\right\}^{\eta}+\frac{1}{V_{\infty}} \int_{A_{\sigma \eta}} \mathbf{n}_{\eta \varpi} C_{\eta}^{\prime} d A\right)\right]-\frac{1}{V_{\infty}} \int_{A_{\varpi \eta}} \mathbf{n}_{\eta \varpi} \cdot\left(\langle\mathbf{u}\rangle_{\eta}\langle C\rangle^{\eta}-\varepsilon_{\eta} \mathbf{D}_{\eta} \cdot \nabla\langle C\rangle^{\eta}\right) d A
\end{aligned}
$$

Under the assumption of large-scale equilibrium: $\left\{\langle C\rangle^{\eta}\right\}^{\eta}=\left\{\langle C\rangle^{\sigma}\right\}^{\varpi}=\{\langle C\rangle\}$ (Ahmadi et al., 1998) and $\varphi_{\eta}\left\{\langle\mathbf{u}\rangle_{\eta}\right\}^{\eta}+\varphi_{\sigma}\left\{\langle\mathbf{u}\rangle_{\sigma}\right\}^{\sigma}=\{\langle\mathbf{u}\rangle\}$ (Whitaker, 1999), the two equations can be added. The assumption of large-scale equilibrium is commonly invoked, as the length scale of $\eta$ and $\omega$ is often considered to be small in comparison to the averaging volume. This assumption will be revisited and relaxed in the adopted implementation by calculating $\{\langle\mathbf{u}\rangle\}$ and $\{\langle C\rangle\}$ numerically as discussed in the next section. Recall that $\langle\mathbf{u}\rangle, \mathbf{u}^{\prime},\langle C\rangle$, and $C^{\prime}$ denote the fine-scale velocity and concentration components, the inter-region flux terms cancel out (because the normal vectors are in opposite directions): 


\section{Averaged equation assuming large-scale equilibrium:}

$$
\begin{aligned}
& \bar{\varepsilon} \frac{\partial\{\langle C\rangle\}}{\partial t}+\nabla \cdot\left(\varphi_{\eta}\left\{\mathbf{u}_{\eta}^{\prime} C_{\eta}^{\prime}\right\}^{\eta}+\varphi_{\sigma}\left\{\mathbf{u}_{\sigma}^{\prime} C_{\sigma}^{\prime}\right\}^{\sigma}\right)+\{\langle\mathbf{u}\rangle\} \cdot \nabla\{\langle C\rangle\}= \\
& \nabla \cdot\left[\left(\varphi_{\eta} \varepsilon_{\eta} \mathbf{D}_{\eta}+\varphi_{\sigma} \varepsilon_{\sigma} \mathbf{D}_{\sigma}\right) \cdot \nabla\{\langle C\rangle\}\right]+\nabla \cdot\left[\frac{\varepsilon_{\eta} \mathbf{D}_{\eta}}{V_{\infty}} \cdot \int_{A_{\pi \eta}} \mathbf{n}_{\eta \sigma} C_{\eta}^{\prime} d A+\frac{\varepsilon_{\sigma} \mathbf{D}_{\sigma}}{V_{\infty}} \cdot \int_{A_{\sigma \eta}} \mathbf{n}_{\varpi \eta} C_{\varpi}^{\prime} d A\right]
\end{aligned}
$$

Deviation equation assuming large-scale equilibrium (subtracting Eq. 7 from Eq. 1):

$$
\begin{aligned}
& \varepsilon^{\prime} \frac{\partial C^{\prime}}{\partial t}-\nabla \cdot\left(\varphi_{\eta}\left\{\mathbf{u}_{\eta}^{\prime} C_{\eta}^{\prime}\right\}^{\eta}+\varphi_{\sigma}\left\{\mathbf{u}_{\sigma}^{\prime} C_{\sigma}^{\prime}\right\}^{\sigma}\right)+\langle\mathbf{u}\rangle \cdot \nabla C^{\prime}+\mathbf{u}^{\prime} \cdot \nabla\{\langle C\rangle\} \\
& =\nabla \cdot\left[\left(\varphi_{\eta} \varepsilon_{\eta} \mathbf{D}_{\eta}+\varphi_{\sigma} \varepsilon_{\sigma} \mathbf{D}_{\sigma}\right) \cdot \nabla C^{\prime}\right]-\nabla \cdot\left[\frac{\varepsilon_{\eta} \mathbf{D}_{\eta}}{V_{\infty}} \cdot \int_{A_{\sigma \eta}} \mathbf{n}_{\eta \sigma} C_{\eta}^{\prime} d A+\frac{\varepsilon_{\sigma} \mathbf{D}_{\sigma}}{V_{\infty}} \cdot \int_{A_{\pi \eta}} \mathbf{n}_{\varpi \eta} C_{\varpi}^{\prime} d A\right]
\end{aligned}
$$

The quantity $\varepsilon^{\prime}$ denotes the deviation from the volume average $\bar{\varepsilon}$. Most upscaling schemes assume steady-conditions; therefore, the transient terms are set to be zero. The steady-state forms of Eqs. 7 and 8 are obtained:

$$
\begin{aligned}
& \nabla \cdot\left(\varphi_{\eta}\left\{\mathbf{u}_{\eta}^{\prime} C_{\eta}^{\prime}\right\}^{\eta}+\varphi_{\sigma}\left\{\mathbf{u}_{\pi}^{\prime} C_{\sigma}^{\prime}\right\}^{\sigma}\right)+\{\langle\mathbf{u}\rangle\} \cdot \nabla\{\langle C\rangle\}=\nabla \cdot\left[\left(\varphi_{\eta} \varepsilon_{\eta} \mathbf{D}_{\eta}+\varphi_{\sigma} \varepsilon_{\sigma} \mathbf{D}_{\sigma}\right) \cdot \nabla\{\langle C\rangle\}\right]+\alpha_{\sigma \eta} \\
& \underbrace{-\nabla \cdot\left(\varphi_{\eta}\left\{\mathbf{u}_{\eta}^{\prime} C_{\eta}^{\prime}\right\}^{\eta}+\varphi_{\sigma}\left\{\mathbf{u}_{\sigma}^{\prime} C_{\sigma}^{\prime}\right\}^{\sigma}\right)+\langle\mathbf{u}\rangle \cdot \nabla C^{\prime}+\mathbf{u}^{\prime} \cdot \nabla\{\langle C\rangle\}}_{\mathrm{LHS}_{\mathrm{Eq}, 10}}=\underbrace{\nabla\left[\left(\varphi_{\eta} \varepsilon_{\eta} \mathbf{D}_{\eta}+\varphi_{\sigma} \varepsilon_{\sigma} \mathbf{D}_{\sigma}\right) \cdot \nabla C^{\prime}\right]-\alpha_{\sigma \eta} \ldots}_{\mathrm{RHS}_{\mathrm{Eq}, 10}}
\end{aligned}
$$

where $\alpha_{\omega \eta}$ denotes summation of the two inter-regional flux terms in Eqs. 7 and 8. Eqs. 9 and 10 can be used to estimate the averaged scaled-up parameters at a snapshot of time under quasisteady-state conditions. Rearranging Eq. 9 and combining all the terms that represent mass transfer due to diffusion, dispersion and inter-regional transport into an effective mass transfer flux (Kechagia et al., 2002):

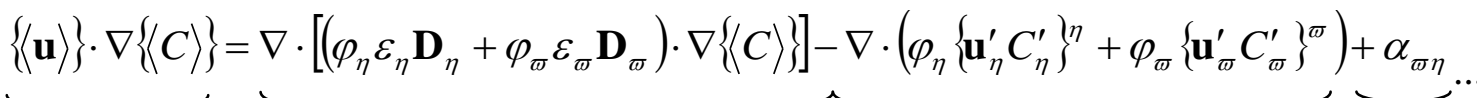

$$
\begin{aligned}
& \underbrace{}_{\text {convection }} \\
& \text { large-scale diffusion } \\
& \text { dispersion } \\
& \text { inter-regional transport } \\
& \{\langle\mathbf{u}\rangle\} \cdot \nabla\{\langle C\rangle\}=K_{e f f}\left(C_{\max }-\{\langle C\rangle\}\right)
\end{aligned}
$$


$K_{\text {eff }}$ is the effective mass transfer coefficient, representing the combined effects of mass transfer due to diffusion, dispersion and inter-regional transport. This equation is valid for the transport of passive (non-reactive) solute particles. In the case of injection, $C_{\max }$ in Eq. $11 \mathrm{~b}$ is taken to be the solute concentration at injection conditions. It is shown in previous works that the scaling characteristics of $K_{\text {eff }}$ directly determine the scaling characteristics of transport response (Leung, 2009, 2014; Leung \& Srinivasan, 2012).

It should be noted that the averaged and deviation equations - Eqs. 9 and 10 - are coupled. Eq. 10 is typically solved by imposing a "closure condition" such that the summation of the average and deviation quantities satisfies the global mass balance condition. (Kechagia et al., 2002): In the conventional implementation, fine-scale solutions to the flow and transport problems are not explicitly known, but the two equations can be decoupled with the following assumptions: 1) arithmetic averaging of $\langle\mathbf{u}\rangle$ and $\langle C\rangle$; and 2) average concentration and its gradient are independent. Under these assumptions, the average concentration and the perturbation can be solved for independently, although the resultant complicated mathematical formulations can be at best applied to mostly simple geometries such as a unit cell containing a periodic medium (Wood, 2013). In the following, an alternative approach based on the availability of fine-scale quantities is presented.

\section{Method}

In this approach, fine-scale quantities $\langle\mathbf{u}\rangle$ and $\langle C\rangle$ obtained from numerical simulation are incorporated into an optimization procedure to update the values of $\{\langle C\rangle\}$ and $C^{\prime}$ until a converged solution to Eqs. 9 and 10 is reached (Leung, 2009; Leung \& Srinivasan, 2012). The procedure is to first perform numerical flow simulation over a representative small portion of the 
reservoir depicting the underlying heterogeneity and obtain the fine-scale quantities. Next, simulations are performed at different coarse scales using reservoir models obtained via flowbased upscaling techniques (e.g. local upscaling scheme that involves solution of the steady-state pressure equation). This second step would yield the coarse-scale quantities $\{\langle\mathbf{u}\rangle\}$ and $\{\langle C\rangle\}$, relaxing the assumptions of arithmetic averaging and large-scale equilibrium that are invoked in the original volume averaging formulations. However, since the boundary conditions in the flowbased upscaling might not be exactly identical to the fine-scale problem, if $\{\langle C\rangle\}$ is substituted back into Eqs. 9 and 10 directly, there would most likely be a slight mismatch between the two sides of the equations. Therefore, $\{\langle C\rangle\}$ is adjusted iteratively using a non-linear optimization scheme such that the difference between the left and right hand sides of Eqs. 9 and 10 are minimized. A major advantage for incorporating flow simulation results is that it facilitates application of the volume averaging techniques to realistic geologic medium, as opposed to those periodic media employed in conventional formulations.

Knowledge of the fine-scale solution relaxes many of the restrictive assumptions associated with the traditional volume averaging formulations. The procedure allows the deviation and averaged equations to remain coupled. Furthermore, as in the conventional approach, the velocity terms, $\{\langle\mathbf{u}\rangle\}$ and $\mathbf{u}^{\prime}$ will be obtained separately from the momentum balance.

In the implementation of this proposed approach, the continuum fine-scale quantities are defined at the grid resolution of the fine-scale flow simulation. The objective is to obtain effective transport parameters (e.g., $K_{\text {eff }}$ ) for a number of coarse-scale simulation grid blocks, which may consist of regions $\omega, \eta$ in various proportions. It should be emphasized that region definition can be stochastic, as long as the proportion of each region within the averaging scale 
(i.e., coarse-scale grid block) is known.

In cases where the length scale of heterogeneity is larger than the averaging (grid block) scale, the assumption of stationary rock-type proportions within an averaging volume at least the size of representative elementary volume (REV), is not exact. Applying any averaging scheme at scales smaller than REV introduces variability in the corresponding averaged attribute (e.g., $K_{\text {eff) }}$. In such cases, the averaged quantity would be characterized by a probability distribution, whose variance is a measure of the variability of the spatial average at that particular length scale (Leung \& Srinivasan, 2011; Vishal \& Leung, 2015). This variance decreases with averaging scale and is considered negligible at REV (Lake \& Srinivasan, 2004). In this work, volume averaging is implemented as a framework to couple coarse-scale averages and fine-scale deviations. A probability distribution of $K_{\text {eff }}$ values is obtained by pooling the results from all the coarse-scale grid blocks. This procedure is repeated at multiple averaging scales. At the end, the probability distribution (uncertainty) of $K_{\text {eff }}$ at different averaging scales can be calibrated. Once these probability distributions are calibrated, they facilitate the assignment of $K_{\text {eff }}$ at the coarse scale by (1) sampling from the probability distribution calibrated for the appropriate averaging scale, (2) assigning the corresponding value of $K_{\text {eff }}$ to a particular coarse-grid location, and (3) repeating steps 1-2 for all coarse-grid locations. Under the assumption of spatial stationarity, the probability distribution of $K_{\text {eff }}$ computed over a small domain is considered to be representative elsewhere. If large-scale non-stationarity (trend) is present, probability distributions of $K_{\text {eff }}$ should be estimated over numerous sub-regions.

\section{Implementation Details}

Since the inter-regional integrals appear in both the averaged and deviation equations, 
explicit calculation of these terms is unnecessary; instead, they should be evaluated together as “ $\alpha_{\omega \eta} "$. Key steps are outlined below:

1. Generate a fine-scale heterogeneous reservoir model (porosity and permeability) for a section of the reservoir and subject to flow simulation to obtain $\langle\mathbf{u}\rangle$ and $\langle C\rangle$. This is referred to as the simulation domain in Figure 1.

2. Perform flow-based upscaling of reservoir permeability at various coarse scales and subject each to flow simulation to obtain $\{\langle\mathbf{u}\rangle\}$ and initial estimates of $\{\langle C\rangle\}$ or $\{\langle C\rangle\}^{\text {initial }}$ at different coarse scales. Note that these are termed initial estimate since the boundary conditions applied during flow-based upscaling might not be exactly identical to the fine-scale problem.

3. Choose a sub-domain for volume averaging calculations to avoid boundary effects due to wells (Figure 1). It is common practice to retain a fine grid around wells in order to accurately represent the dynamic of flow in the vicinity of the well.

4. At a given snapshot of time $t$ and a particular averaging scale, make perturbations to $\{\langle C\rangle\}$ using a non-linear minimization scheme, such that the left and right hand sides of equations (9) and (10) are matched. A global optimization method of the Very Fast Simulated Annealing (VFSA) is implemented due to its computational efficiency (Sen \& Stoffa, 2013). First, using $\{\langle C\rangle\}^{\text {initial }}$, compute $C^{\prime}$, evaluate $\alpha_{\omega \eta}$ with Eq. 9 and substitute the result into Eq. 10. Second, calculate err representing the mismatch between the left- and right-hand sides of Eq. 10: err $=\left\|L H S_{E q .10}-R H S_{E q .10}\right\|$. Based on a pre-defined acceptance/rejection criterion, perturb $\{\langle C\rangle\}$ and propose $\{\langle C\rangle\}^{\text {new }}$. Third, repeat the step until the convergence criterion is reached.

5. Compute $K_{\text {eff }}$ at each coarse-scale grid cell by equating the RHS of Eq. 11a with the RHS of 
Eq. 11b. A probability distribution of $K_{\text {eff }}$ is constructed by aggregating its corresponding value from all coarse-scale grid blocks.

6. Repeat Steps 4-5 for other averaging length scales and values of $t$.

7. The procedure yields a set of probability distributions of $K_{\text {eff }}$ at different length scales corresponding to different times during the flow and transport process. These characteristics will be for regions that are not in the immediate vicinity of wells.

\section{Case Studies and Discussion}

\section{Case Study 1 - Comparison Between Covariance Model and Channelized Model}

The transport of a passive solute in a 500×500 2-D fine grid model is simulated (Figure 1). The grid blocks are of uniform size $(\Delta x=\Delta y=10 \mathrm{~m})$ with a thickness of $10 \mathrm{~m}$. Injection and production rates are held constant at $300 \mathrm{~m}^{3} /$ day. Solute particles are added to the injection stream at a concentration of $1 \mathrm{mg} / \mathrm{m}^{3}$, with diffusion coefficient $=1 \times 10^{-6} \mathrm{~m}^{2} /$ day. Numerical dispersion is controlled in the flow simulation by implementing a higher-order finite-difference approximation scheme. Two synthetic models are constructed (Figure 2): Case 1 - a covariance model generated using SISIM (Deutsch \& Journel, 1992) and Case 2 - a channelized model generated using SNESIM (Remy et al., 2009). The following semivariogram is fitted to the channelized model, and it is subsequently used to generate the covariance model. Therefore, both models are constrained to the same first- and second-order moments; hence they are expected to exhibit similar uncertainty distributions at least up to the bivariate distributions:

- Model parameters of the semivariogram $\chi(\mathbf{h})$ :

Nugget effect $=0$ 
- Structure \#1: spherical model, sill $=0.18, a_{\max 1}=650 \mathrm{~m} ; a_{\min 1}=170 \mathrm{~m}$;

$\circ \quad$ Structure \#2: exponential model, sill $=0.82$; range $a_{\max 2}=720 \mathrm{~m} ; a_{\min 2}=230 \mathrm{~m}$.

- The lithofacies proportion is $72 \%$ for facie 1 and $28 \%$ for facie 2 .

The subscripts max and min represent the directions of maximum (azimuth $=90^{\circ}$ ) and minimum (azimuth $=0^{\circ}$ ) anisotropy, while $\boldsymbol{h}$ and $a$ denote lag distance and range, respectively. Formulae for the spherical model and exponential models can be found in Journel \& Hujbregts (1978).

Results at 25000 days (0.33 injected pore volumes) are used for scale-up calculations. It is clear from the corresponding particle breakthrough profiles in Figure 3 that the total mass transfer is higher for the channelized model after 25000 days. Figure 4 shows the maps of permeability and magnitude of velocity over the volume averaging domain for a coarse grid size of 10x10. Significant smoothing is observed comparing the magnitude of average velocity $\{<\mathbf{u}>\}$ to that of the fine-scale velocity $<\mathbf{u}>$. Figure 5 shows the corresponding concentration maps. These figures also show the spatial distribution of the perturbed quantities: $\mathbf{u}^{\prime}$ and $C^{\prime}$. For the case of covariance model, the map of concentration deviations does not show a crisp outline delineating the different heterogeneous regions. The summation of the concentration deviations over the entire volume averaging domain is close to zero as a result. Highest concentration deviations $\left(C^{\prime}\right)$ are localized along the fluid front. On the other hand the deviation map for the channelized system shows a clear demarcation of the underlying heterogeneity. Figure 6 shows the spatial distribution of absolute value of $K_{\text {eff, }}$ whose values are the highest in regions that are swept by and along the front, while being negligible ahead of the front. This is because the maximum amount of mass transfer between the injection fluid and the in-situ fluid occurs over areas that are contacted by the particles. Higher magnitude of $K_{\text {eff }}$ are observed in the channelized case because the convective transport is strongly aligned to the direction of heterogeneity. Larger 
variance in $K_{\text {eff }}$ is also observed since a strong contrast in concentration values is maintained between the high permeability (high velocity) areas and low permeability areas.

Figure 7 and Figure 8 show the spatial mean and standard deviation of the absolute value of $K_{\text {eff, }}$ mass fluxes due to dispersion, convection, and diffusion (corresponding to each term in Eq. 11a) as a function of averaging scale. With the exception of diffusion, all functions exhibit higher mean and variance in the channelized model. This decreasing trend with scale is expected because as the length scale increases, transport is restricted to a few pathways; a large fraction of the domain remains uncontacted, rendering mass transfer to be less efficient. Diffusion, however, is controlled by local concentration gradient; hence, it is less influenced by large-scale heterogeneous features. The slower decline in $K_{\text {eff }}$ variance suggests that the length scale over which REV is attained is larger for the channelized model.

A strong correspondence between $K_{\text {eff }}$ and breakthrough behavior has been observed. In particular, higher variability in breakthrough behavior can be expected in models with longer correlated features oriented along the direction of flow (Leung \& Srinivasan, 2012). The fluid would seek the fastest flow path, and this is evident when long correlated features are oriented in the flow direction; the fastest flow path, however, would vary among different realizations of the reservoir properties, especially if the correlation length is long. On the other hand, when the correlation length is short, fluid would tend to disperse away from the advection front and delay breakthrough, but the variability in breakthrough behavior among different realizations of reservoir properties would be more subdued due to substantial smearing of particles away from the front in all realizations. As a result, higher variability in $K_{\text {eff }}$ and breakthrough behavior is observed for the channelized model.

The results also reveal that the corresponding transport behavior could still differ, despite 
being constrained to the same univariate and bivariate statistics. It is conceivable that over a small domain (e.g., a $3 \times 3$ grid or 30m x 30m) extracted within a particular facie, the difference between the two models would be minimal; as a result, the corresponding transport response would also be similar. However, if these results are scaled-up to a much larger volume, ignoring high-order multivariate distribution could potentially underestimate the ensuing uncertainties in $K_{\text {eff, as }}$ the results in Figure 7 indicate that both the mean and variance in $K_{\text {eff }}$ are lower for the covariance model. Therefore, characterization of high-order multivariate distribution is an important consideration.

Variation of $K_{\text {eff }}$ with the volume of investigation is also assessed using multiple realizations. Fifty fine-scale realizations $(\Delta x=\Delta y=10 \mathrm{~m})$ are generated for each of the four different domain sizes: $10 \times 10,25 \times 25,50 \times 50$ and $100 \times 100$. The procedure is repeated for both the covariance and channelized cases; hence, a total of 400 sub-models of the original $500 \times 500$ models are constructed. All realizations are subjected to the aforementioned transport simulation. The injection/production is prorated according to the total volume of each sub-model to preserve the local pressure gradient/velocity ratio. A value $K_{\text {eff }}$ is computed for each model using Eq. $11 \mathrm{~b}$ with an average convective flux and concentration difference. Figure 9 shows the mean of $K_{\text {eff }}$ over 50 realizations as a function of domain size, and the trends are in good agreement with those observed in Figure 7.

The computing code was implemented with MATLAB® (MathWorks, 2009). It took approximately 6 minutes to execute 300 iterations for each length scale using the University of Alberta's Mathematical and Statistical Servers. Therefore, a total of 30 minutes were needed to generate the results shown in Figure 7. In all experiments, the reduction in error/mismatch tended to stabilize in fewer than 300 iterations. 
The relative importance of physical dispersion $\left(D_{\text {physical }}\right)$ in comparison to numerical dispersion $\left(D_{\text {num }}\right)$ is assessed next. Lantz (1971) presented the expression of dimensionless numerical dispersivity $\left(\alpha_{\text {num }}\right)$ corresponding to the 1D transport equation, while a generalized numerical dispersivity tensor for a multidimensional system was derived by Fanchi (1983). Analyzing both formulations reveal that the maximum value of $\alpha_{\text {num }}$ in 1D or maximum diagonal element in the dimensionless numerical dispersivity tensor in 3D can be computed using Eq. 12, where $\Delta \xi$ and $\Delta \tau$ are dimensionless grid block size and dimensionless time step, respectively (Lantz, 1971).

$$
\alpha_{\text {num }}=\frac{\Delta \xi+\Delta \tau}{2} \text { where } \Delta \xi=\frac{\Delta x}{L} \quad \Delta \tau=\frac{\Delta t_{\max } u}{\phi L}
$$

$\Delta x$ and $\Delta t_{\max }$ denote the grid size and maximum time step, while $L$ and $u$ represent the domain size and average flow velocity, respectively. The contribution of $\Delta \tau$ is negligible, since the steady-state forms of Eqs. 9 and 10 are employed. Isolating the term corresponding to dispersive flux from Eq. 11a, an expression for $D_{\text {physical }}$ can be defined as follows:

$$
D_{\text {phyiscal }} \nabla^{2}\{\langle C\rangle\}=\nabla \cdot\left(\varphi_{\eta}\left\{\mathbf{u}_{\eta}^{\prime} C_{\eta}^{\prime}\right\}^{\eta}+\varphi_{\varpi}\left\{\mathbf{u}_{\sigma}^{\prime} C_{\varpi}^{\prime}\right\}^{\varpi}\right)
$$

The right-hand-side of Eq. 13 is identical to the second term in Eq. 11a, representing mass transfer due to field-scale dispersion in the coarse-scale models. The dimensionless total dispersivity $\alpha_{\text {total }}$ is now defined as:

$$
\alpha_{\text {total }}=\frac{\left(D_{\text {diffusion }}+D_{\text {physical }}\right) \phi}{L u}
$$

An average value of $D_{\text {physical }}$ is estimated at each averaging scale, and, as an example, the corresponding values of $\alpha_{\text {num }}$ and $\alpha_{\text {total }}$ for the covariance model are compared in Table 1. Although that numerical dispersion cannot be fully isolated from the calculation $D_{\text {physical }}$, the 
comparison illustrates that, except for the extremely large averaging scale, $\alpha_{\text {num }}$ is smaller than

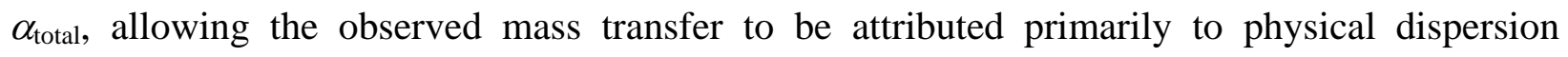
instead of numerical dispersion.

\section{Case Study 2 - Scaling Characteristic of Fractal Media}

In this section, the scaling behavior obtained with the volume averaging approach is compared to other published analytical results for fractal media. Using the program "Hydro_gen" (Bellin \& Rubin, 1996), two unconditional self-affine (fractal) random space functions (RSF) are generated. Spatial distribution of porosity is obtained by re-scaling the generated RSF values to range approximately between 0.05 and 0.2 (with a mean of 0.126 ), while permeability values are computed based on an empirical function: $k(\mathrm{mD})=1408000 \phi^{3.8}$ (Nelson, 1994), with values ranging approximately between 15 to $3000 \mathrm{mD}$ (with a mean of $600 \mathrm{mD}$ ). Porosity and permeability maps for the two cases with different $H$ values are shown in Figure 10, where $H$ is the Hurst dimension and is related to the fractal dimension (DF):

$$
\mathrm{DF}=2-H
$$

Histograms of porosity and permeability distributions for the two cases are also shown in Figure 10. Experimental semivariograms, which follow the power-law model of $\chi(h) \sim h^{2 H}$ (Journel \& Hujbregts, 1978), are computed for the two cases and displayed in Figure 11.

Particles are injected and produced at $200 \mathrm{~m}^{3} / \mathrm{d}$ in the 500x500 2-D fine grid fractal medium (Figure 1), with all other parameters set to be the same as Case Study 1. Results at 60000 days (0.38 injected pore volumes similar to Case 1) are used for scale-up calculations. Maps of permeability and magnitude of velocity over the volume-averaging domain for a coarse grid size of 10x10 are illustrated in Figure 12. Figure 13 shows the corresponding concentration maps. 
Figure 14 shows the spatial distribution of absolute value of $K_{\text {eff. }}$

For a fractal media, dispersivity $\left(\alpha_{L}\right)$ increases with scale of observation $(L)$ in the following manner (Arya, Hewett, Larson, \& Lake, 1988; Mandelbrot \& Van Ness, 1968; Sahimi, 1993) :

$$
\alpha_{L} \propto L^{3-2 D F} \propto L^{2 H-1} \text { where } \mathrm{DF}=2-H \text { is defined as the fractal dimension. }
$$

Since the dominant mechanism is dispersion, it is conjectured that the following relationship of $K_{\text {eff }}$ would apply:

$$
K_{e f f} \propto D^{*} \frac{\partial^{2} C}{\partial x^{2}} \propto \alpha_{L} u \frac{\partial^{2} C}{\partial x^{2}} \propto\left(\frac{\alpha_{L}}{L}\right)\left(u \frac{\partial C}{\partial x}\right)
$$

where $D^{*}$ is the dispersion coefficient. Given that the field-scale injection/production rate is kept constant, $u \partial C / \partial x$ is constant in Eq. 17 ; hence, $K_{\text {eff }}$ should vary with $\alpha_{L} / L$. Figure 15 shows variation in $K_{\text {eff }}$ as a function of scale. The values of $\alpha_{L} / L$ or $L^{2 \mathrm{H}-1} / L$ (based on Eqs. 15-16) against $L$ is also plotted for comparison. Therefore, it is inferred that $K_{\text {eff }}$ varies with scale in a fashion similar to $\alpha_{L} / L$. This ratio of $\alpha_{L} / L$ is equivalent to the inverse of Peclet number, which represents a dimensionless mixing length (Perkins \& Johnston, 1963). Results in Figure 15 indicate that the scaling characteristics of $K_{\text {eff }}$ and dispersion obtained with the volume averaging approach are in good agreement with the analytical predictions based on the fractal theory for dispersion-dominant transport. Scale-dependent dispersion increases with value of $H$, enhancing the effective mass transfer coefficient $\left(K_{e f f}\right)$ at larger scales, as evidenced by the increasingly flattening decline of $K_{\text {eff }}$ with $L$ when $H=0.8$ in Figure 15 . With $H=0.55$ (higher fractal dimension), increased overall mass transfer due to dispersion and convection (Figure 16) is observed. The variation in spatial mean and standard deviation of the absolute values of convection and diffusion with scale shown in Figure 16 also verifies that process is controlled by convection and dispersion, since the local diffusive flux is quite insignificant. This result 
demonstrates the difference in scaling behavior of effective mass transfer coefficient due to fractal dimension.

\section{Conclusions and Future Work}

1. A quantitative approach to quantify the variation and uncertainty of effective mass transfer coefficient with scale in heterogeneous reservoirs is implemented. The volume averaging approach is modified by utilizing information from numerical fine-scale flow simulations and integrating heterogeneity into the scale-up of transport equations. We begin with governing equations at the fundamental continuum or Darcy scale and proceed with spatial averaging in order to derive the averaged equations at the local heterogeneity scale.

2. An original contribution of this work is to provide an insight into how the scaling of effective mass transfer coefficient is influenced by particular heterogeneity distributions. The results suggest that various aspects of heterogeneity descriptions would have a strong impact on the ensuing transport behavior. In addition to the univariate (histogram) statistics, continuity of spatially-varying properties as characterized by the higher-order multivariate statistics could also considerably influence the scale-up results.

3. Results presented in this work emphasize that proper scale-up of heterogeneity to the field scale is necessary when one attempts to scale-up transport response from results obtained over a much smaller lab or pilot scale. The impacts of higher-order multivariate distributions and large-scale heterogeneity are typically not captured in small-scale experiments. Ignoring their impacts could severely underestimate the ensuing uncertainties in $K_{\text {eff }}$ and mass transfer.

4. Though the results obtained in this study are specific to the model set-up used in the case 
study, the presented averaging approach is general and can be applied to quantify the effect of spatial variability on effective transport parameters in other reservoir settings. This work also highlights the importance of adopting a modeling framework that takes into consideration the uncertainty in heterogeneity description.

5. It can be argued that scaling analysis of $K_{\text {eff }}$ would provide only a qualitative assessment of heterogeneity impacts on solute transport, as direct application of $K_{\text {eff }}$ in existing commercial simulation tools is not well defined. A possible avenue for future work is to estimate from Eqs. 13 and 14 scaled-up dispersivity values, which can be incorporated in practical fieldscale simulation. This work illustrates the feasibility of volume averaging method in deriving coarse-scale transport equations and estimating parameters such as $K_{\text {eff }}$ and $\alpha_{\text {total }}$ at different scales.

\section{Nomenclature}

$A_{\omega \eta}=$ area of the boundary between $\eta$ and $\omega$-regions contained with the averaging volume, $\mathrm{L}^{2}$

$a_{\max }=$ correlation range in the major direction of anisotropy, $\mathrm{L}, \mathrm{m}$

$a_{\min }=$ correlation range in the minor direction of anisotropy, $\mathrm{L}, \mathrm{m}$

$C=$ solute concentration, $\mathrm{m} / \mathrm{L}^{3}, \mathrm{mg} / \mathrm{m}^{3}$

$\langle C\rangle^{\omega},\langle C\rangle^{\eta}=$ concentration at local continuum scale in $\omega$ or $\eta$-region, $\mathrm{m} / \mathrm{L}^{3}, \mathrm{mg} / \mathrm{m}^{3}$

$\left\{\langle C\rangle^{\omega}\right\}^{\omega},\left\{\langle C\rangle^{\eta}\right\}^{\eta}=$ intrinsic average concentration over the $\omega$ or $\eta$-region, $\mathrm{m} / \mathrm{L}^{3}, \mathrm{mg} / \mathrm{m}^{3}$

$\left\{\langle C\rangle^{\omega}\right\},\left\{\langle C\rangle^{\eta}\right\}=$ superficial average concentration over the $\omega$ or $\eta$-region $\mathrm{m} / \mathrm{L}^{3}, \mathrm{mg} / \mathrm{m}^{3}$

$C_{\omega}^{\prime}, C_{\eta}^{\prime}=$ spatial deviation of $\langle C\rangle^{\omega}$ or $\langle C\rangle^{\eta}, \mathrm{m} / \mathrm{L}^{3}, \mathrm{mg} / \mathrm{m}^{3}$

$\{\langle C\rangle\}=\langle C\rangle-C^{\prime}=\left\{\langle C\rangle^{\eta}\right\}^{\eta}=\left\{\langle C\rangle^{\omega}\right\}^{\omega}=$ intrinsic average concentration at large-scale equilibrium, $\mathrm{m} / \mathrm{L}^{3}, \mathrm{mg} / \mathrm{m}^{3}$

$C_{\max }=$ maximum concentration, $\mathrm{m} / \mathrm{L}^{3}, \mathrm{mg} / \mathrm{m}^{3}$

$D_{\text {diffusion }}=$ diffusion, $\mathrm{L}^{2} / \mathrm{t}, \mathrm{m}^{2} / \mathrm{d}$

$D_{\text {physical }}=$ physical dispersion, $\mathrm{L}^{2} / \mathrm{t}, \mathrm{m}^{2} / \mathrm{d}$

$D_{\text {num }}=$ numerical dispersion, $\mathrm{L}^{2} / \mathrm{t}, \mathrm{m}^{2} / \mathrm{d}$ 
$\mathbf{D}_{\omega}, \mathbf{D}_{\eta}=$ effective dispersion tensors at the local continuum scale in the $\omega$ or $\eta$-region, $\mathrm{L}^{2} / \mathrm{t}, \mathrm{m}^{2} / \mathrm{d}$ $D F=$ fractal dimension

$H=$ Hurst exponent

$k=$ permeability, $\mathrm{L}^{2}, \mathrm{mD}$

$K_{\text {eff, }}=$ effective mass transfer coefficient of solvent in phase $j, \mathrm{t}^{-1}, \mathrm{~d}^{-1}$

$L=$ domain size, $\mathrm{L}, \mathrm{m}$

$l=$ heterogeneity length scale, $\mathrm{L}, \mathrm{m}$

$t=$ time, $\mathrm{t}, \mathrm{d}$

$\Delta t=$ time step, $\mathrm{t}, \mathrm{d}$

$u=$ average flow velocity, $\mathrm{L} / \mathrm{t}, \mathrm{m} / \mathrm{d}$

$\langle\mathbf{u}\rangle_{\omega},\langle\mathbf{u}\rangle_{\eta}=$ velocity at the local continuum scale in the $\omega$ or $\eta$-region, $\mathrm{L} / \mathrm{t}, \mathrm{m} / \mathrm{d}$

$\left\{\langle\mathbf{u}\rangle_{\omega}\right\}^{\omega},\left\{\langle\mathbf{u}\rangle_{\eta}\right\}^{\eta}=$ intrinsic average velocity over the $\omega$ or $\eta$-region, $\mathrm{L} / \mathrm{t}, \mathrm{m} / \mathrm{d}$

$\left\{\langle\mathbf{u}\rangle_{\omega\}},\left\{\langle\mathbf{u}\rangle_{\eta}\right\}=\right.$ superficial average velocity over the $\omega$ or $\eta$-region, $\mathrm{L} / \mathrm{t}, \mathrm{m} / \mathrm{d}$

$\mathbf{u}_{\omega}^{\prime}, \mathbf{u}_{\eta}^{\prime}=$ spatial deviation of $\langle\mathbf{u}\rangle_{\omega},\langle\mathbf{u}\rangle_{\eta}, \mathrm{L} / \mathrm{t}, \mathrm{m} / \mathrm{d}$

$\{\langle\mathbf{u}\rangle\}=\langle\mathbf{u}\rangle-\mathbf{u}^{\prime}=\varphi_{\eta}\left\{\langle\mathbf{u}\rangle_{\eta}\right\}^{\eta}+\varphi_{\omega}\left\{\langle\mathbf{u}\rangle_{\omega}\right\}^{\omega}=$ superficial average velocity at large-scale equilibrium,

$\mathrm{L} / \mathrm{t}, \mathrm{m} / \mathrm{d}$

$V_{\infty}=$ averaging volume, $\mathrm{L}^{3}$

$\mathbf{n}_{\omega \eta}=$ normal vector of the boundary between the $\eta$ and $\omega$-regions

$\mathrm{x}, \mathrm{y}, \mathrm{z}=$ refers to coordinate directions $\mathrm{x}, \mathrm{y}, \mathrm{z}$

$\Delta x=$ grid block size, $\mathrm{L}, \mathrm{m}$

\section{Greek letters:}

$\alpha_{L}=$ longitudinal dispersivity, $\mathrm{L}, \mathrm{m}$

$\alpha_{\text {num }}=$ numerical dispersivity, dimensionless

$\alpha_{\text {total }}=$ physical dispersivity plus diffusion, dimensionless

$\Delta \xi=$ dimensionless grid block size

$\Delta \tau=$ dimensionless time step

$\varepsilon_{\omega}, \varepsilon_{\eta}=$ total porosity in the $\omega$ or $\eta$-region, fraction

$\bar{\varepsilon}=$ large-scale average porosity, fraction

$\varepsilon^{\prime}=$ deviation of $\varepsilon_{\omega}$ and $\varepsilon_{\eta}$, fraction 
$\phi=$ porosity $[=] \mathrm{L}^{3} / \mathrm{L}^{3}$, fraction

$\chi(\mathbf{h})=$ semivariogram or variogram at a lag distance of $\mathbf{h}$

$\varphi_{\omega}, \varphi_{\eta}=$ volume fraction of the $\omega$ or $\eta$-region, fraction

*Dimension notations: $L$ is length, $m$ is mass, $t$ is time

*bolded quantities refer to vector or matrix

\section{Acknowledgments}

This research was partially supported by the U.S. Department of Energy, Office of Science,

Office of Basic Energy Sciences. The Center for Frontiers of Subsurface Energy Security (CFSES) is a DOE Energy Frontier Research Center, under Contract No. DE-SC0001114. This

research has been enabled by the use of computing resources provided by WestGrid and Compute/Calcul Canada. The authors would also like to thank Schlumberger Ltd., who provided the academic licenses for ECLIPSE, and the University of Alberta for granting access to the Numerical and Statistical Server.

\section{References}

Ahmadi, A., Quintard, M., \& Whitaker, S. (1998). Transport in chemically and mechanically heterogeneous porous media: V. two-equation model for solute transport with adsorption. Advances in Water Resources, 22(1), 59-86.

Arya, A., Hewett, T., Larson, R., \& Lake, L. (1988). Dispersion and reservoir heterogeneity. SPE Reservoir Engineering, 3(1), 139-148.

Brenner, H. (1980a). Dispersion resulting from flow through spatially periodic porous media. Philosophical Transactions of the Royal Society (London). 297, 81-133.

Brenner. H. (1980b). A general theory of Taylor dispersion phenomena. Physiochemical Hydrodynamics, 1, 91-123. 
Chrysikopoulos, C. V., Kitanidis, P. K., \& Roberts, P.V. (1992). Macrodispersion of sorbing solutes in heterogeneous porous formations with spatially periodic retardation factor and velocity field. Water Resources Research, 28(6), 1517-1529.

Chrysikopoulos, C. V. (1995). Effective parameters for flow in saturated heterogeneous porous media. Journal of Hydrology, 170(1), 181-197.

Coutelieris, F. A., Kainourgiakis, M. E., Stubos, A. K., Kikkinides, E. S., \& Yortsos, Y. C. (2006). Multiphase mass transport with partitioning and inter-phase transport in porous media. Chemical Engineering Science, 61(14), 4650-4661.

Cushman, J. H. (1982). Proofs of the volume averaging theorems for multiphase flow. Advances in Water Resources, 5(4), 248-253.

Cvetkovic, V., \& Gotovac, H. (2014). On the upscaling of chemical transport in fractured rock, Water Resources Research, 50, 5797-5816.

Deutsch, C. V., \& Journel, A.G. (1992). GSLIB: Geostatistical Software Library and User's Guide, Oxford University Press, New York, NY, 340 pp.

Fanchi, J.R. (1983). Multidimensional numerical dispersion. SPE Journal, 23(1), 143-151.

Fathi, E., \& Akkutlu, I. Y. (2012). Mass transport of adsorbed-phase in stochastic porous medium with fluctuating porosity field and nonlinear gas adsorption kinetics. Transport in Porous Media, 91(1), 5-33.

Howes, F. A., \& Whitaker, S. (1985). The spatial averaging theorem revisited. Chemical Engineering Science, 40(8), 1387-1392.

Journel, A. G., \& Hujbregts, C. (1978). Mining geostatistics. New York City: Academic Press.

Kechagia, P. E., Tsimpanogiannis, I. N., Yortsos, Y. C., \& Lichtner, P. C. (2002). On the upscaling of reaction-transport processes in porous media with fast or finite kinetics. Chemical Engineering Science, 57(13), 2565-2577.

Lake, L. W., \& Srinivasan, S. (2004). Statistical scale-up of reservoir properties: Concepts and applications. Journal of Petroleum Science and Engineering, 44(1-2), 27-39.

Lantz, R.B. (1971). Quantitative evaluation of numerical diffusion (truncation error). SPE Journal, 11(3), 315-320.

Leung, J. Y. (2009). Reservoir modeling accounting for scale-up of heterogeneity and transport processes. (Doctoral dissertation, University of Texas at Austin). 
Leung, J. Y., \& Srinivasan, S. (2011). Analysis of uncertainty introduced by scaleup of reservoir attributes and flow response in heterogeneous reservoirs. SPE Journal, 16(3), 713-724.

Leung, J. Y., \& Srinivasan, S. (2012). Scale-up of mass transfer and recovery performance in heterogeneous reservoirs. Journal of Petroleum Science and Engineering, 86-87(0), 71-86.

Leung, J.Y. (2014). Scaleup of effective mass transfer in vapour-extraction process accounting for field-scale reservoir heterogeneities. Journal of Canadian Petroleum Technology, 53(5), 275-289.

Li, D., \& Lake, L. W. (1995). Scaling fluid flow through heterogeneous permeable media. SPE Advanced Technology Series, 3(1), 188-197.

MathWorks (2009). Version 7.9 (R2009b). Natick, Massachusetts: The MathWorks Inc.

Mandelbrot, B. B., \& Van Ness, J. W. (1968). Fractional brownian motions, fractional noises and applications. SIAM Review, 10(4), 422-437.

Nelson, P. H. (1994). Permeability-porosity relationships in sedimentary rocks. Log Analyst, 35(3), 38-62.

Perkins, T. K., \& Johnston, O. C. (1963). A review of diffusion and dispersion in porous media. SPE Journal, 3(1), 70-84.

Porta, G. M., Chaynikov, S., Thovert, J. F., Riva, M., Guadagnini, A., \& Adler, P. M. (2013). Numerical investigation of pore and continuum scale formulations of bimolecular reactive transport in porous media. Advances in Water Resources, 62, 243-253.

Quintard, M., \& Whitaker, S. (1988). Two-phase flow in heterogeneous porous media: The method of large-scale averaging. Transport in Porous Media, 3(4), 357-413.

Quintard, M., \& Whitaker, S. (1998). Transport in chemically and mechanically heterogeneous porous media IV: Large-scale mass equilibrium for solute transport with adsorption. Advances in Water Resources, 22(1), 33-57.

Remy, N., Boucher, A., \& Wu, J. (2009). Applied geostatistics with SGeMS. $1^{\text {st }}$ ed. Cambridge: Cambridge University Press.

Sahimi, M. (1993). Flow phenomena in rocks: From continuum models to fractals, percolation, cellular automata, and simulated annealing. Reviews of Modern Physics, 65(4), 1393-534.

Sen, M., \& Stoffa, P. (2013). Global optimization methods in geophysical inversion. Cambridge: University Press. 
Lugo-Méndez, H. D., Valdés-Parada, F. J., Porter, M. L., Wood, B. D., \& Ochoa-Tapia, A. (2015). Upscaling diffusion and nonlinear reactive mass transport in homogeneous porous media. Transport in Porous Media, 107(3), 683-716.

Vishal, V., \& Leung, J. Y. (2015). Impacts of subscale heterogeneities on dispersive solute transport in subsurface systems. Journal of Contaminant Hydrology, 182, 63-77.

Wood, B. D. (2009). The role of scaling laws in upscaling. Advances in Water Resources, 32(5), 723-736.

Wood, B.D. (2013). Technical note: Revisiting the geometric theorems for volume averaging. Advances in Water Resources, 62: 340-352.

Whitaker, S. (1999). The method of volume averaging. Dordrecht, The Netherlands: Kluwer Academic.

Zanotti, F., \& Carbonell, R. G. (1984). Development of transport equations for multiphase system-I: General development for two phase system. Chemical Engineering Science, 39(2), 263-278.

Zhang, D., \& Winter, C. L. (1999). Moment-equation approach to single phase fluid flow in heterogeneous reservoirs. SPE Journal, 4(2), 118-127.

\section{Tables}

\begin{tabular}{|c|c|c|}
\hline Averaging scale & $\alpha_{\text {num }}$ & $\alpha_{\text {total }}$ \\
\hline \hline $20 \mathrm{~m} \times 20 \mathrm{~m}$ & $2.00 \mathrm{E}-03$ & $3.69 \mathrm{E}-03$ \\
\hline $50 \mathrm{~m} \times 50 \mathrm{~m}$ & $5.00 \mathrm{E}-03$ & $1.15 \mathrm{E}-02$ \\
\hline $100 \mathrm{~m} \times 100 \mathrm{~m}$ & $1.00 \mathrm{E}-02$ & $1.51 \mathrm{E}-02$ \\
\hline $250 \mathrm{~m} \times 250 \mathrm{~m}$ & $2.50 \mathrm{E}-02$ & $4.81 \mathrm{E}-02$ \\
\hline $500 \mathrm{~m} \times 500 \mathrm{~m}$ & $5.00 \mathrm{E}-02$ & $4.61 \mathrm{E}-02$ \\
\hline
\end{tabular}

Table 1: Comparison of numerical dispersion and physical dispersion for the covariance model in Case Study 1.

\section{Figures}



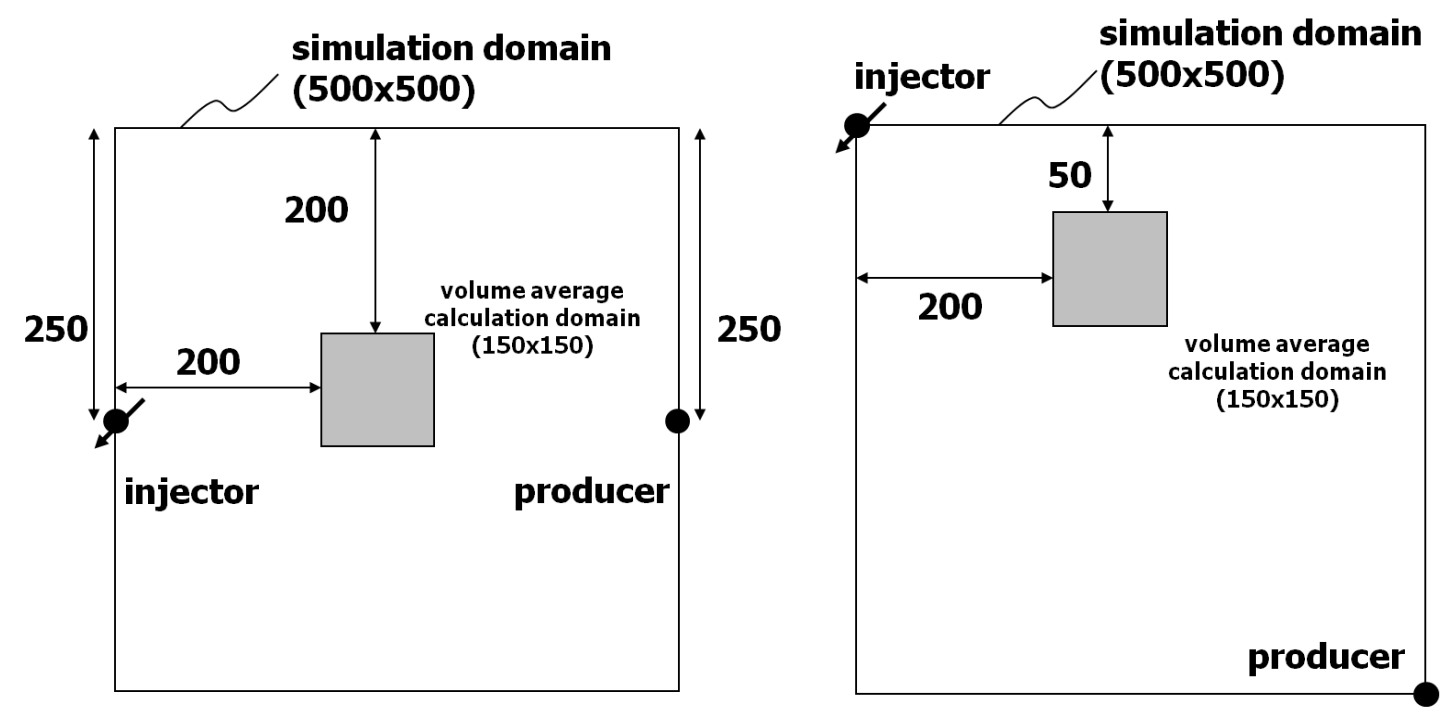

Figure 1: Set-up of simulation and volume averaging calculation domains for the Case Study 1 (left) and Case Study 2 (right).
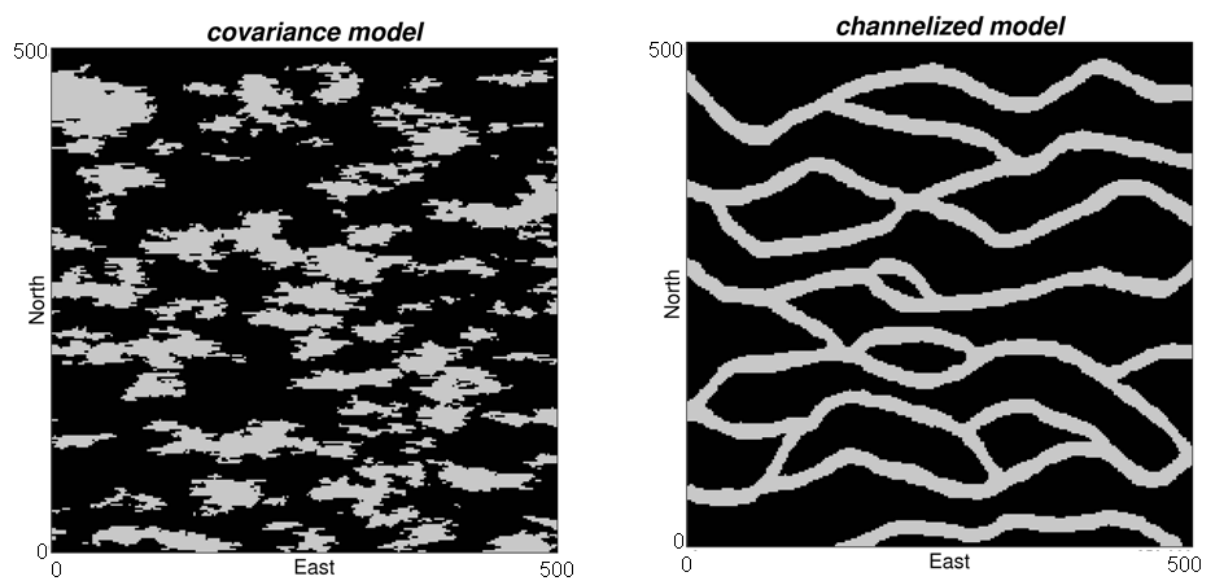

Facie 2

$\phi=0.2$

$\mathrm{k}=3000 \mathrm{md}$

Facie 1

$\phi=0.05$

$\mathrm{k}=15 \mathrm{md}$

Figure 2: Porosity and permeability models for Case Study 1.

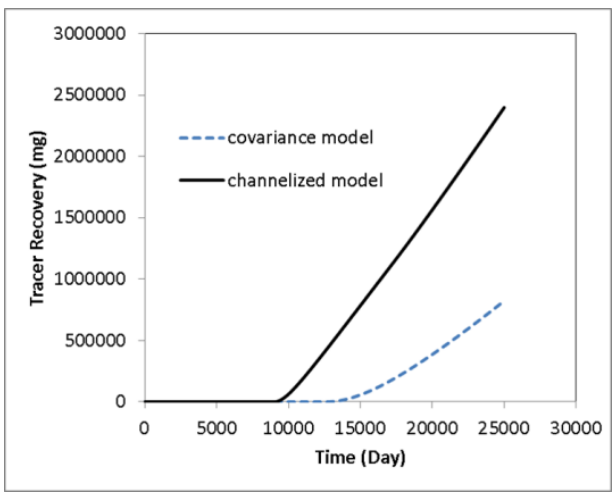

Figure 3: Particle breakthrough profile for Case Study 1. 
covariance model

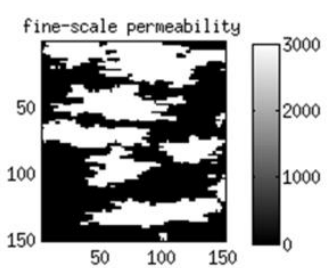

$\{<\mathbf{u}>\}$

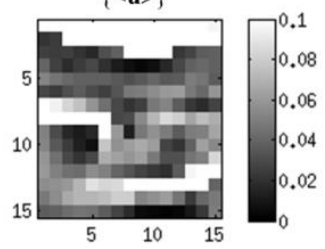

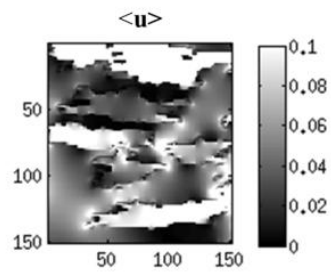

$\mathbf{u}^{\text {' }}$

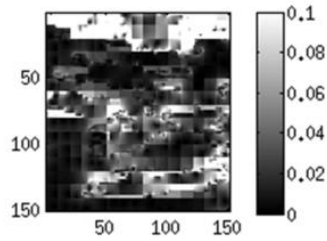

channelized model
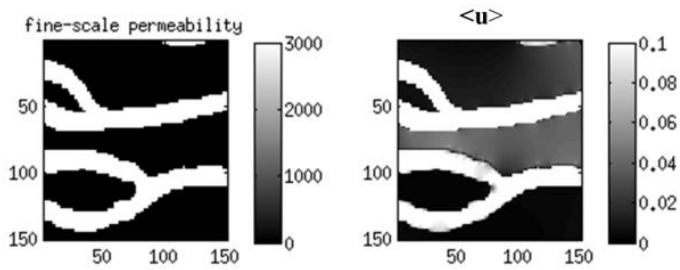

$\mathbf{u}^{\prime}$
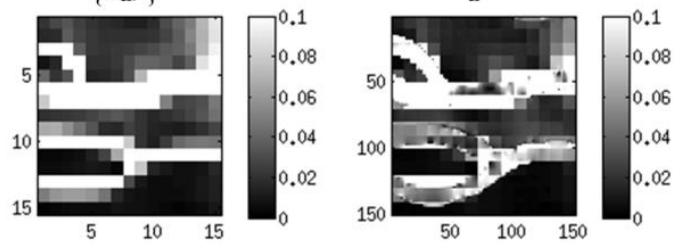

Figure 4: (From top left to bottom right) permeability in $\mathrm{md}$, velocity $<\mathbf{u}>$ in $\mathrm{m} /$ day and its decomposition into average velocity $\{<\mathbf{u}>\}$ and $\mathbf{u}^{\prime}$ for a coarse grid size of $10 \times 10$ in the volume averaging domain (Fig. 1) of Case Study 1.

covariance model
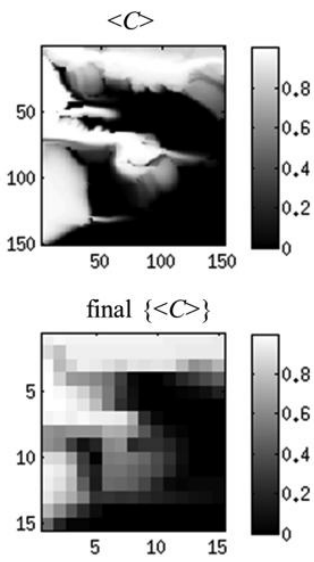

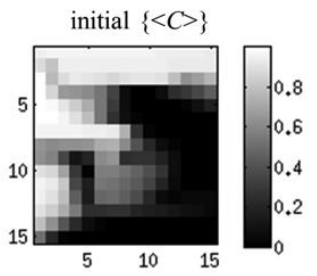

$C^{\prime}$

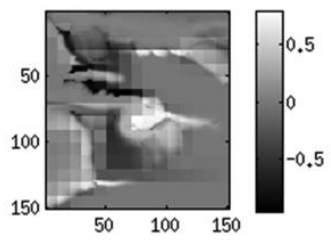

channelized model
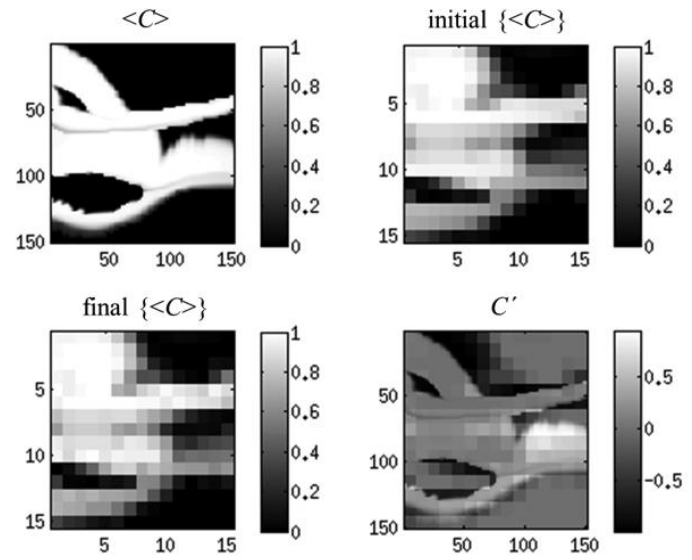

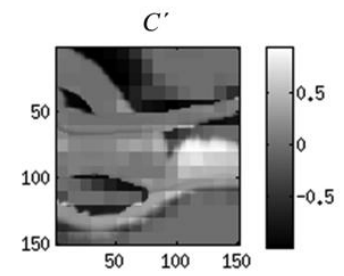

Figure 5: (From top left to bottom right) fine-scale concentration $<C>$ in $\mathrm{mg} / \mathrm{m}^{3}$, average concentration $\{<C>\}$ from coarse-scale simulation (initial guess before simulated annealing), final average concentration $\{<C>\}$ after simulated annealing and the corresponding deviations $C^{\prime}$ for a coarse grid size of $10 \times 10$ in the volume averaging domain (Fig. 1) of Case Study 1. 

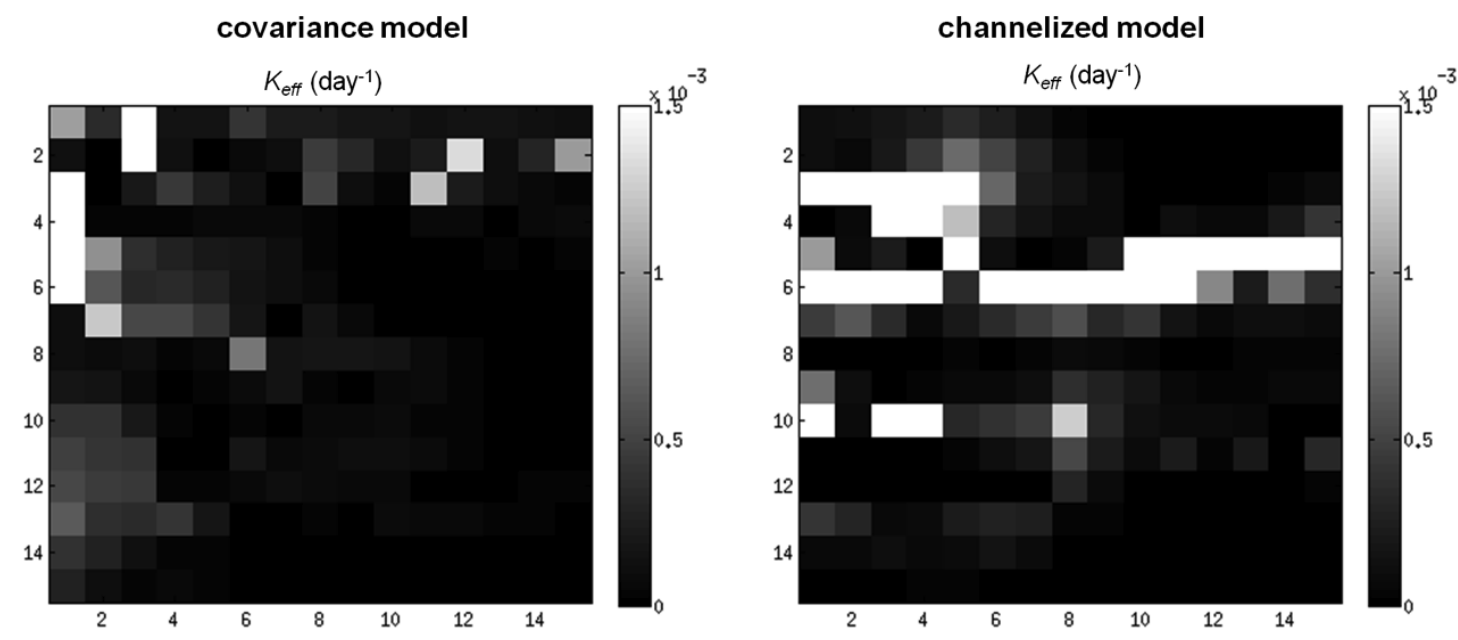

Figure 6: Spatial distribution of $K_{\text {eff }}$ for a coarse grid size of 10x10 in the volume averaging domain (Fig. 1) of Case Study 1. $K_{\text {eff }}$ is the highest in regions that are swept by the front and is close to zero ahead of the front. Higher values of $K_{\text {eff }}$ are observed in the channelized model, reflecting an increase in areas contacted by the concentration front.
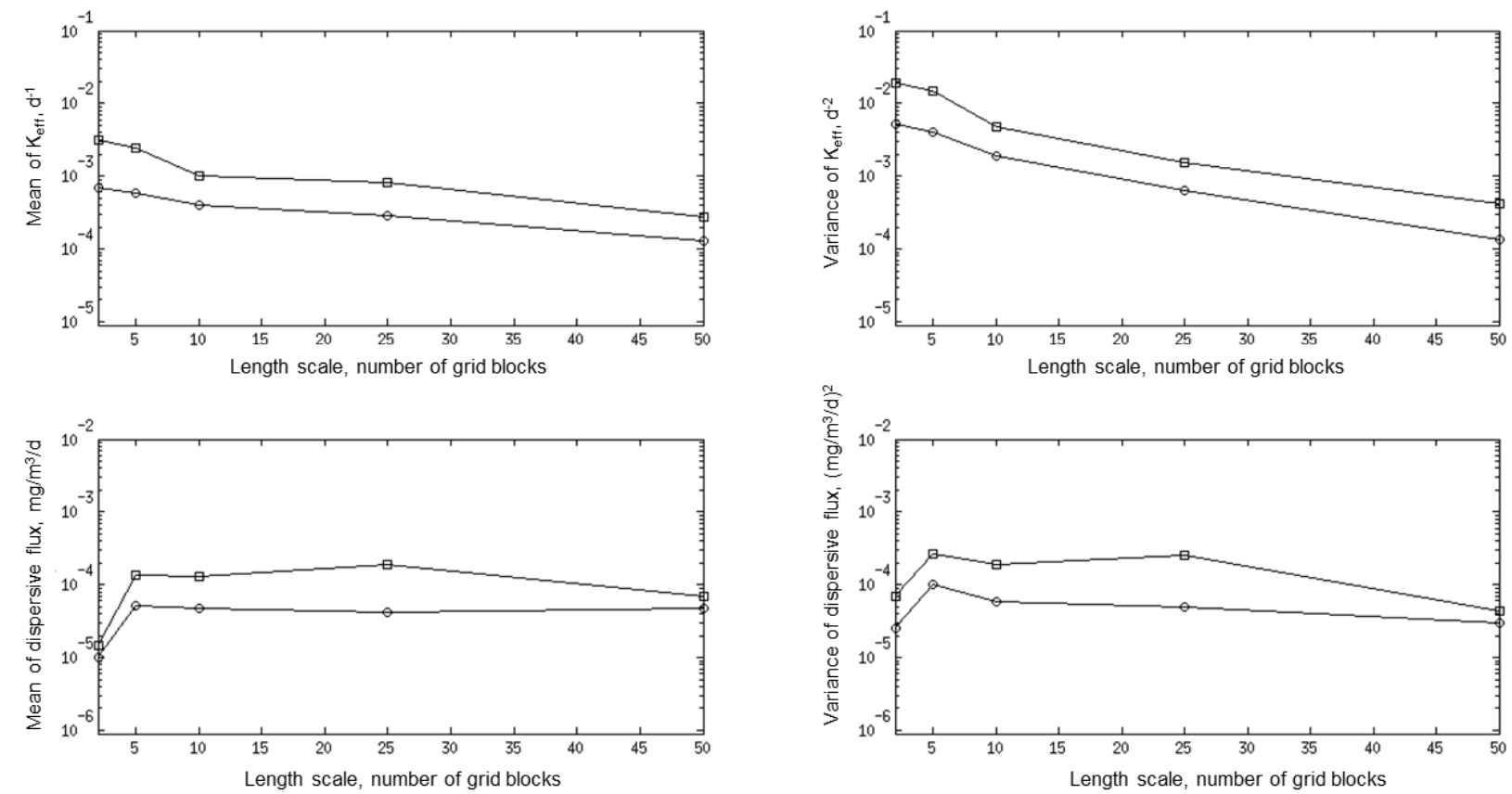

Figure 7: Mean and variance of $K_{\text {eff }}$ and mass transfer due to dispersion as a function of length scale in Case Study 1 (circle: covariance model; square: channelized model). 

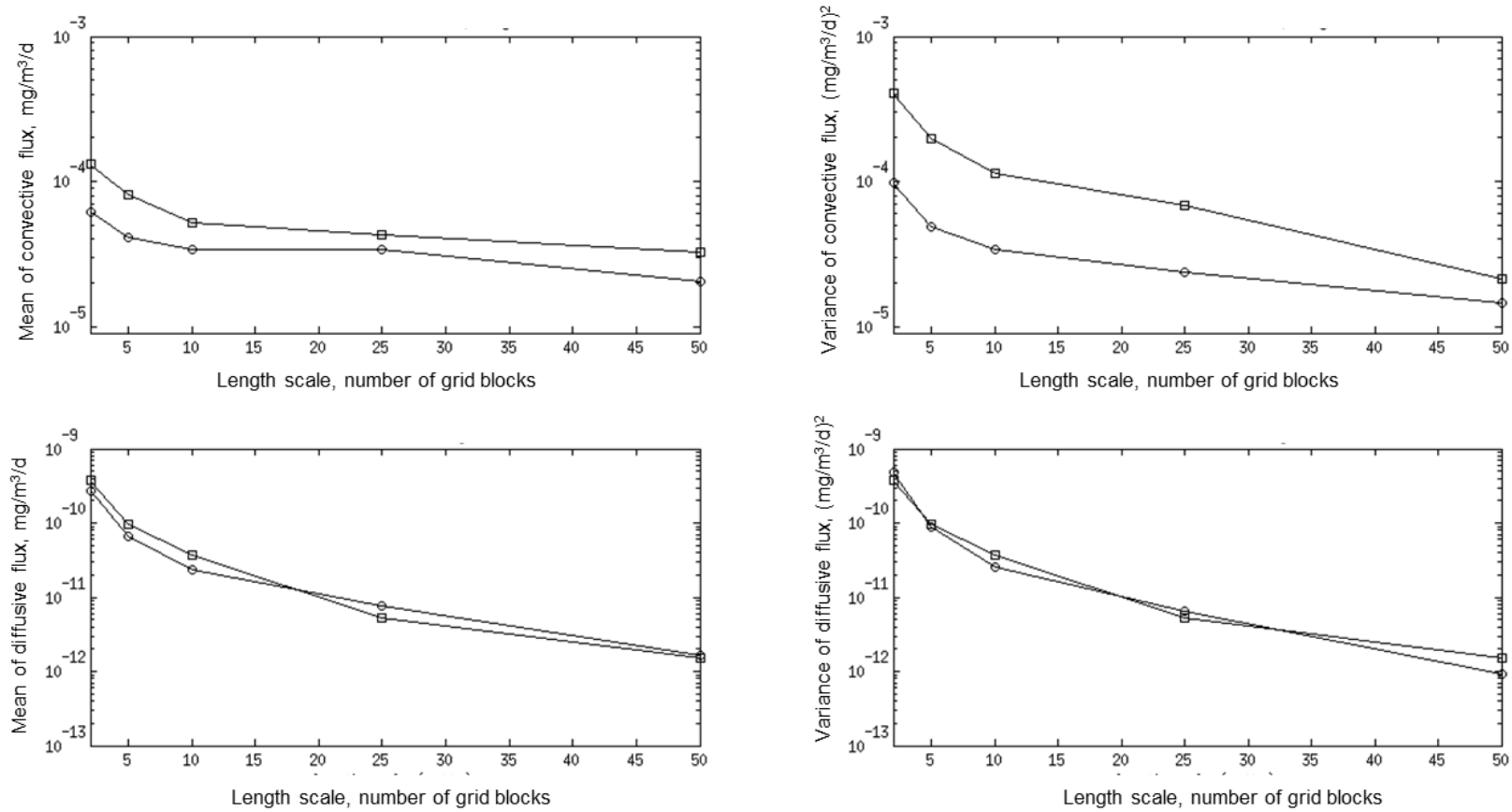

Figure 8: Mean and variance of mass transfer due to convection and diffusion as a function of length scale in Case Study 1 (circle: covariance model; square: channelized model).

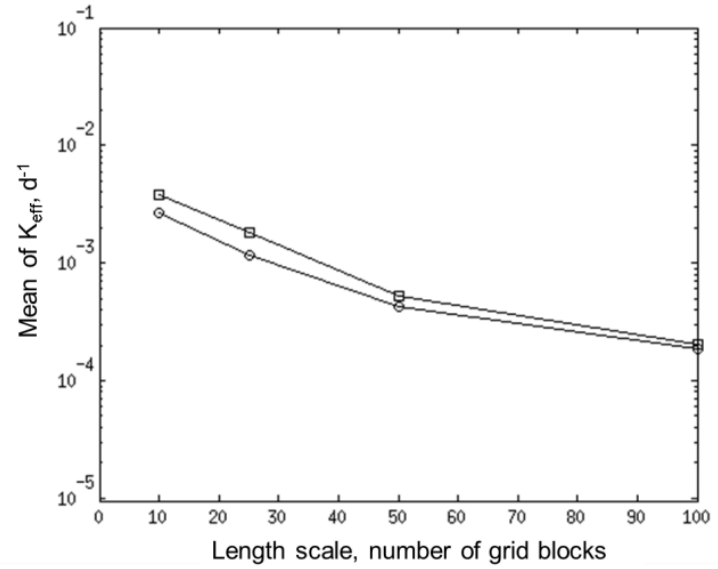

Figure 9: Mean of $K_{\text {eff }}$ computed over 50 realizations for different domain sizes: $10 \times 10,25 \times 25$, $50 \times 50$ and $100 \times 100$ in Case Study 1 (circle: covariance model; square: channelized model). 


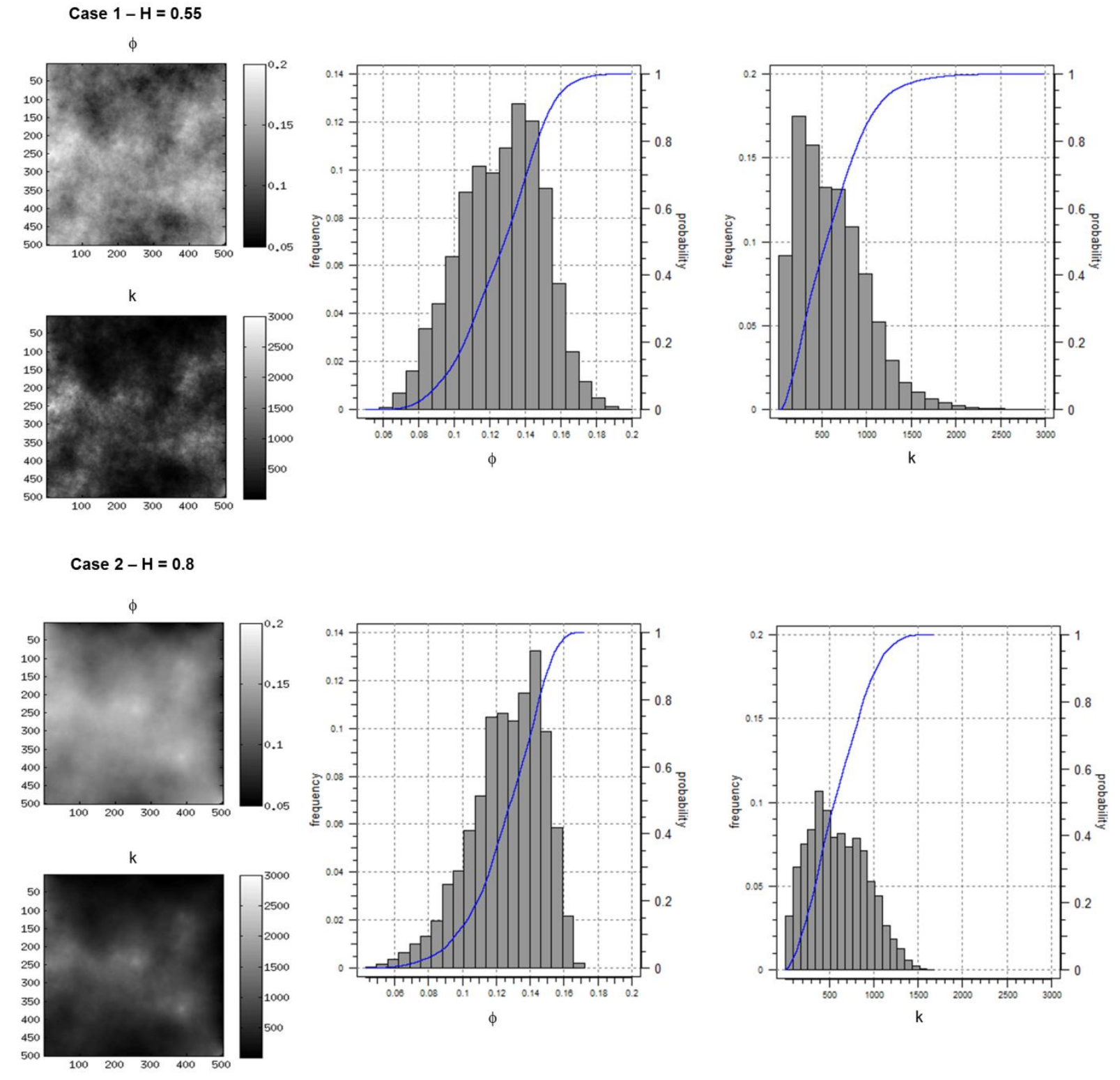

Figure 10: Porosity and permeability models for Case Study 2. 
$\mathrm{H}=\mathbf{0 . 5 5}$

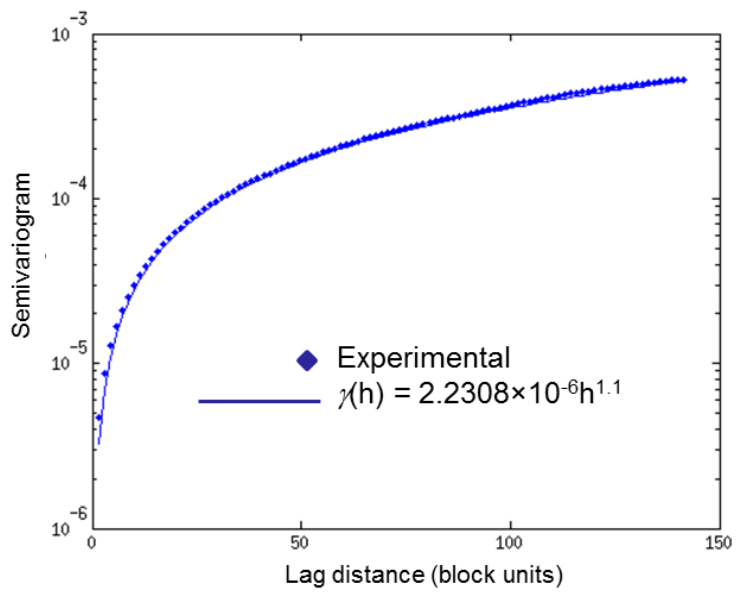

$\mathrm{H}=\mathbf{0 . 8}$

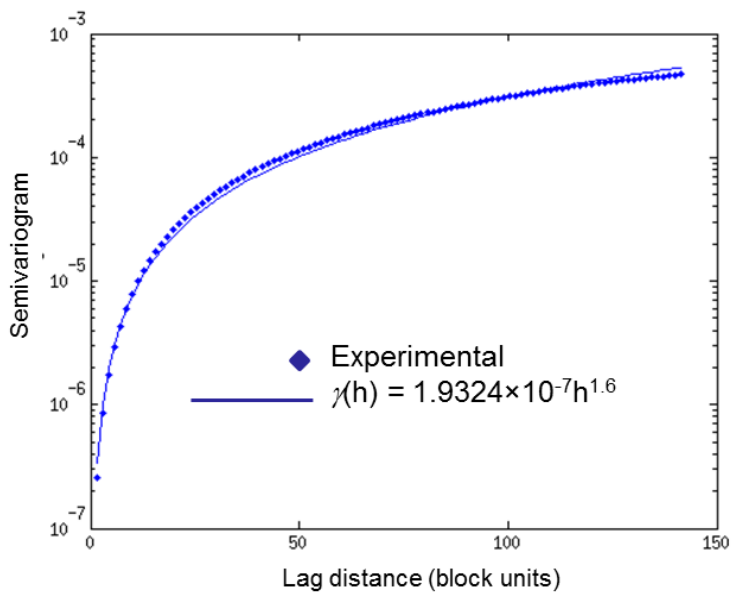

Figure 11: Experimental semivariograms for the two power-law models used in Case Study 2.

$\mathrm{H}=\mathbf{0 . 5 5}$
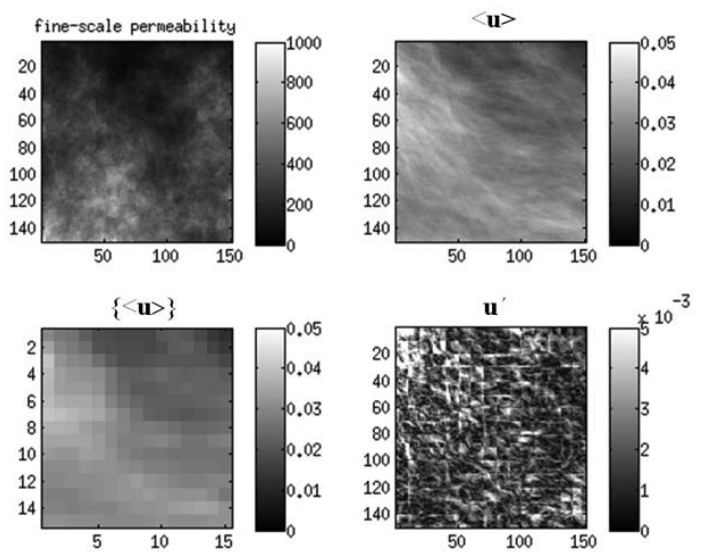

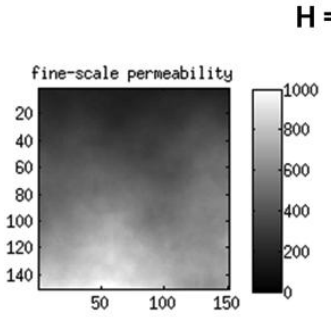

$H=0.8$
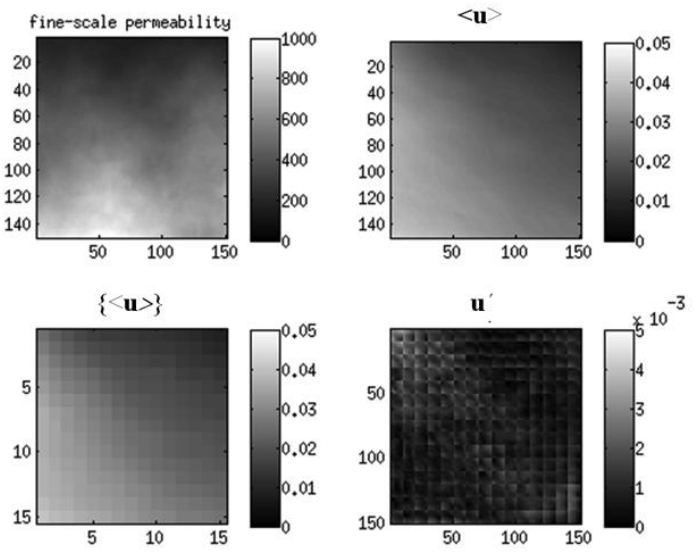

Figure 12: (From top left to bottom right) permeability in $\mathrm{md}$, velocity $<\mathbf{u}>$ in $\mathrm{m} /$ day and its decomposition into average velocity $\{<\mathbf{u}>\}$ and $\mathbf{u}^{\prime}$ for a coarse grid size of $10 \times 10$ in the volume averaging domain (Fig. 1) of Case Study 2. 


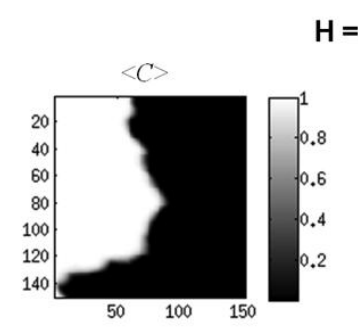

$H=0.55$

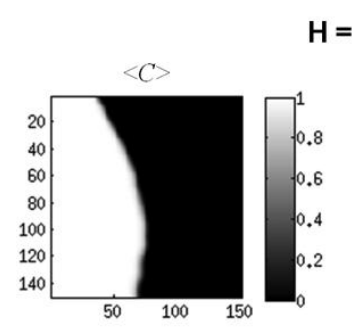

$\mathrm{H}=0.8$
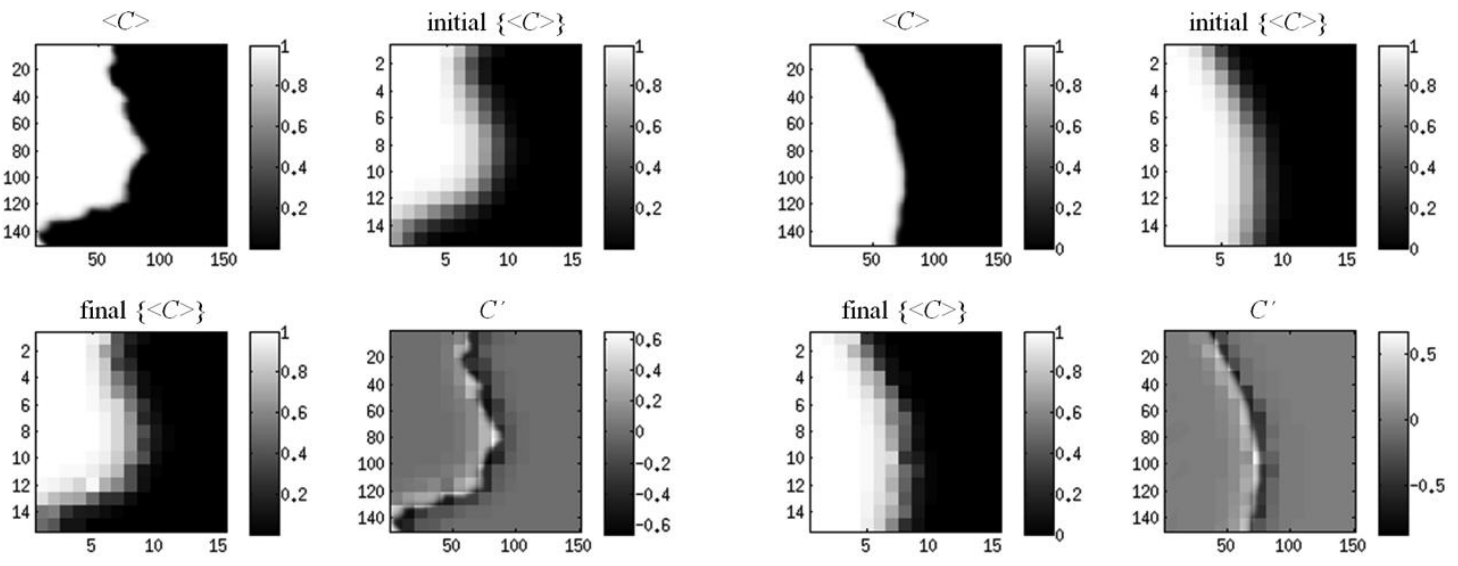

Figure 13: (From top left to bottom right) fine-scale concentration $\langle C\rangle$ in $\mathrm{mg} / \mathrm{m}^{3}$, average concentration $\{\langle C>\}$ from coarse-scale simulation (initial guess before simulated annealing), final average concentration $\{\langle C\rangle\}$ after simulated annealing and the corresponding deviations $C^{\prime}$ for a coarse grid size of $10 \times 10$ in the volume averaging domain (Fig. 1) of Case Study 2.
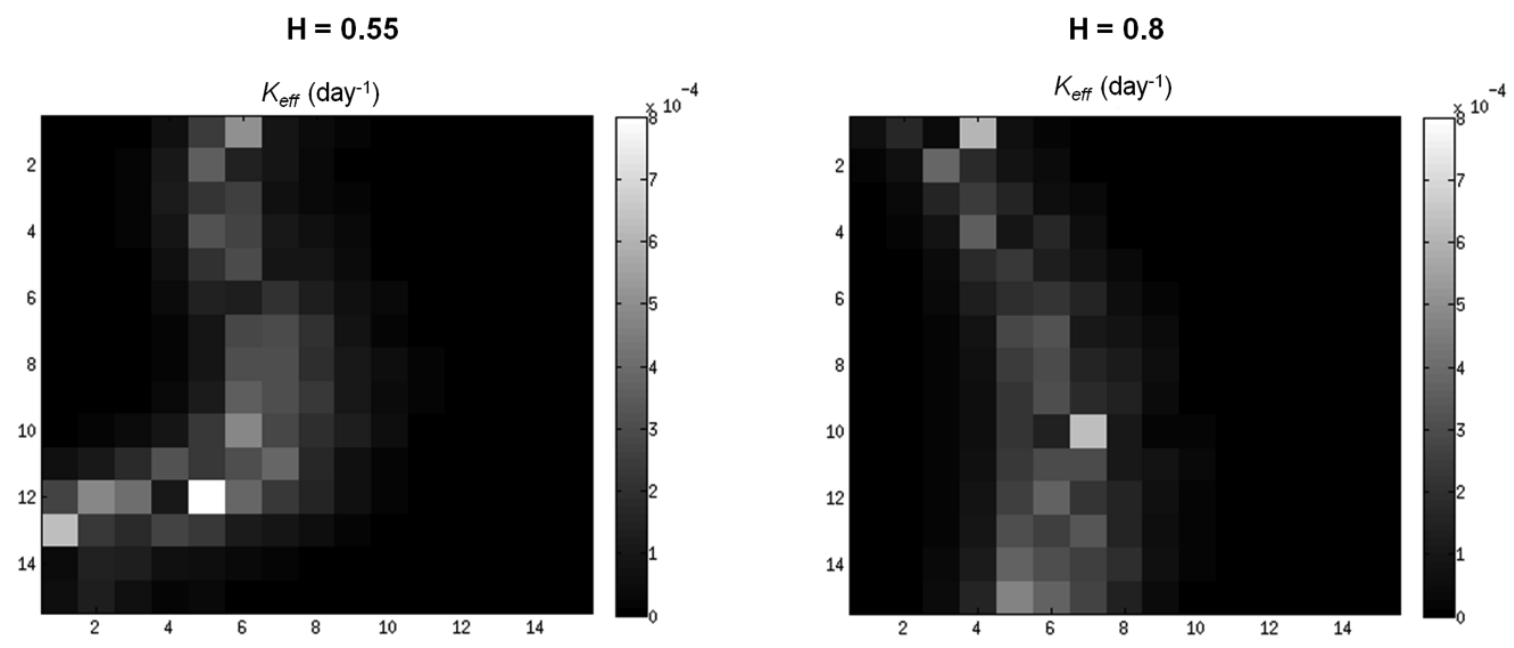

Figure 14: Spatial distribution of $K_{\text {eff }}$ for a coarse grid size of $10 \times 10$ in the volume averaging domain (Fig. 1) of Case Study 2. Higher values of $K_{\text {eff }}$ are observed in the $\mathrm{H}=0.8$ model. 

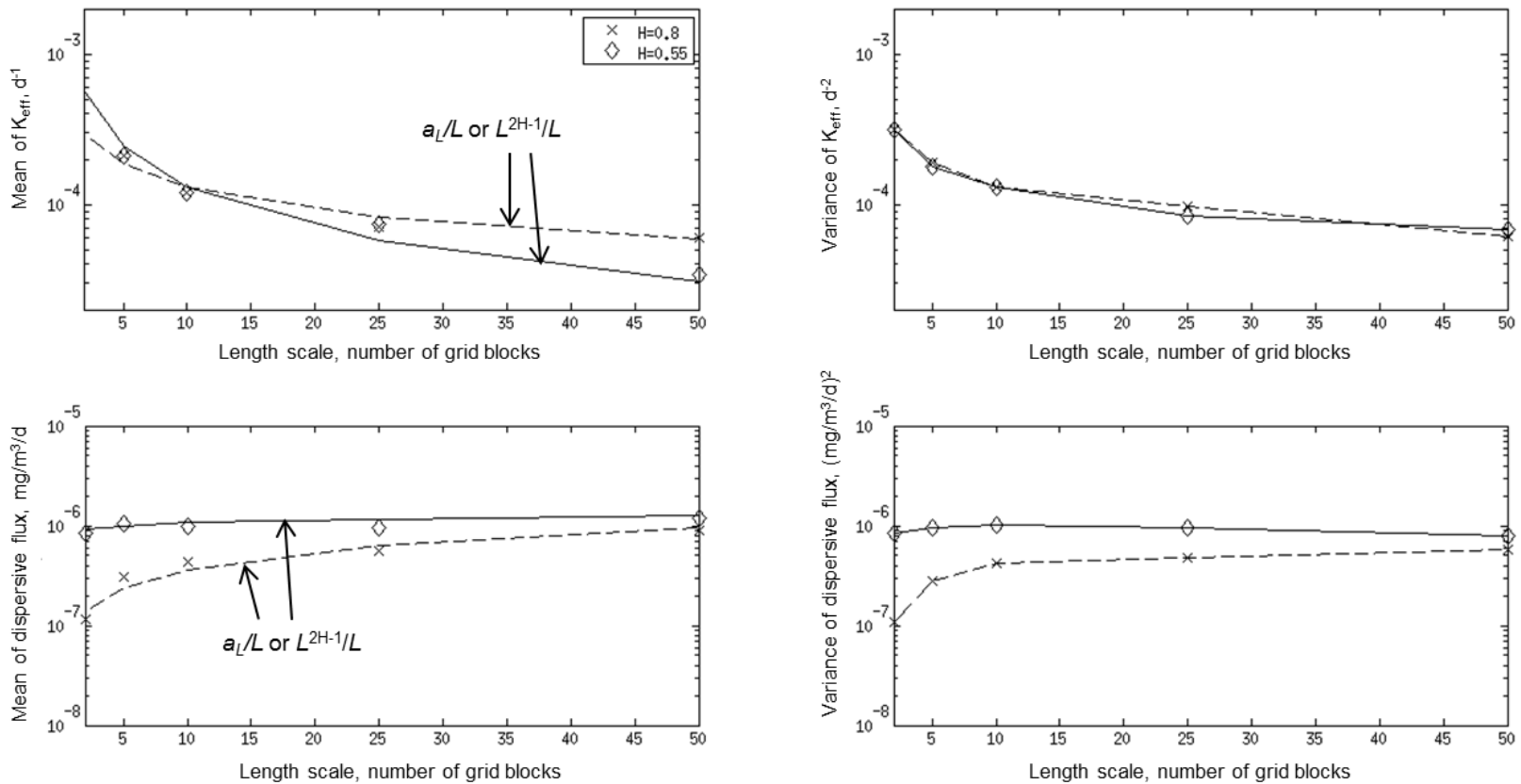

Figure 15: Mean and variance of $K_{\text {eff }}$ and mass transfer due to dispersion as a function of length scale in Case Study 2. The values of $\alpha_{L} / L$ or $L^{2 \mathrm{H}-1} / L$ (based on Eqs. 15-16) against $L$ are shown on the left (diamond and solid line: $\mathrm{H}=0.55$; cross and dashed line: $\mathrm{H}=$ $0.8)$.
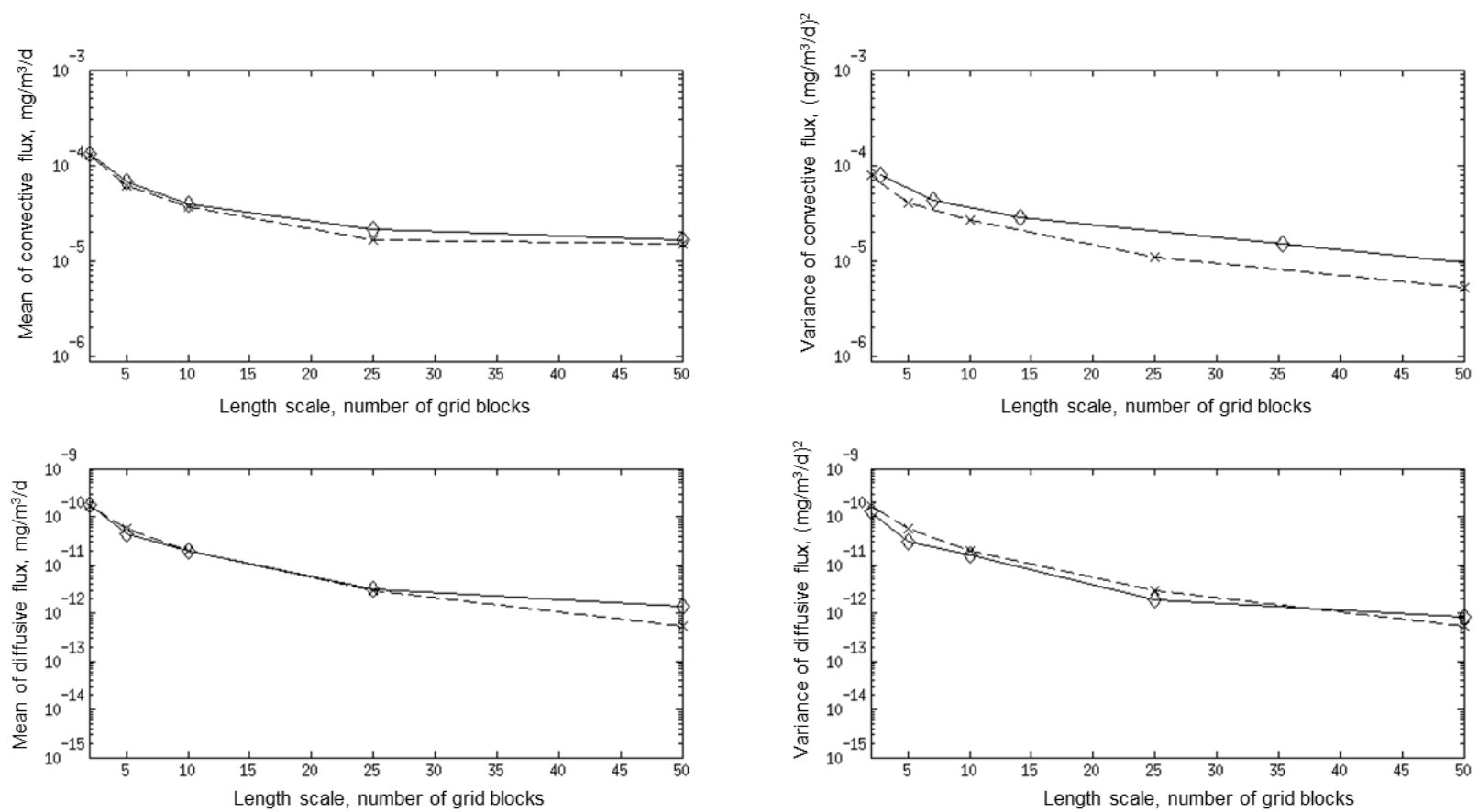

Figure 16: Mean and variance of mass transfer due to convection and diffusion as a function of length scale in Case Study 2 (diamond and solid line: $\mathrm{H}=0.55$; cross and dashed line: $\mathrm{H}=0.8$ ). 